\title{
ASYMPTOTIC PROPERTIES OF THE APPROXIMATE INVERSE ESTIMATOR FOR DIRECTIONAL DISTRIBUTIONS
}

\author{
M. RIPLINGER, ${ }^{*}$ Saarland University \\ M. SPIESS, ${ }^{* *}$ Ulm University
}

\begin{abstract}
For stationary fiber processes, the estimation of the directional distribution is an important task. We consider a stereological approach, assuming that the intersection points of the process with a finite number of test hyperplanes can be observed in a bounded window. The intensity of these intersection processes is proportional to the cosine transform of the directional distribution. We use the approximate inverse method to invert the cosine transform and analyze asymptotic properties of the estimator in growing windows for Poisson line processes. We show almost-sure convergence of the estimator and derive Berry-Esseen bounds, including formulae for the variance.
\end{abstract}

Keywords: Cosine transform; fiber process; inverse problem; Poisson process; rose of intersections; stereology

2010 Mathematics Subject Classification: Primary 60G10

Secondary 60D05

\section{Introduction}

Many porous materials can be modeled with dilated random fiber processes, i.e. processes of dilated curves. While, for the estimation of the intensity of such processes, there already exist some established methods (see, e.g. [13] or [19, Chapter 9]); the estimation of some other properties is still an open problem. We focus on the so-called directional distribution, i.e. the distribution of the tangent direction at a typical point of the fibrous media, and analyze some of the most interesting stochastic properties of the estimator presented in a recent paper by Louis et al. [10].

Consider a stationary random set of fibers $\Xi$ with intensity $\lambda$ and directional distribution density $\varphi$ (with respect to the spherical surface area measure). For the estimation of $\varphi$, we take a stereological approach based on counting the intersections of $\Xi$ with test hyperplanes in an observation window. See [1] for an introduction to stereology.

The essence of the problem can be summarized as follows. For $\xi$ in the unit sphere $S^{d-1}$, denote by $g(\xi)$ the rose of intersections, i.e. the intensity of the process $\Xi \cap \xi^{\perp}$. Then the cosine transform $C$ of the directional distribution $\varphi$ is proportional to $g$, i.e.

$$
g(\xi)=\lambda C \varphi(\xi) .
$$

Received 30 August 2011; revision received 3 May 2012.

* Postal address: Institute of Applied Mathematics, Saarland University, 66041 Saarbrücken, Germany.

Email address: riplinger@num.uni-sb.de

** Postal address: Institute of Stochastics, Ulm University, Helmholtzstr. 18, 89069 Ulm, Germany.

Email address: malte.spiess@uni-ulm.de 
Here, the cosine transform of $\varphi$ is defined as

$$
C \varphi(\xi)=\int_{S^{d-1}}|\langle\xi, v\rangle| \varphi(\nu) \mathrm{d} \nu, \quad \xi \in S^{d-1},
$$

where $\langle\xi, v\rangle$ is the scalar product of $\xi$ and $v$, and $\mathrm{d} v$ denotes integration with respect to the surface area measure. For more information about the cosine transform, see [3].

Now, for some unit vectors $\xi_{1}, \ldots, \xi_{n} \in S^{d-1}$, we assume that the number of intersections of $\Xi$ with the test hyperplanes $\xi_{i}^{\perp}, i=1, \ldots n$, within the observation window $\varrho W=b_{\varrho}(\mathbf{o})$, i.e. the closed ball centered at the origin with radius $\varrho$, are known. This information can be used to estimate the values of $g$ at $\xi_{1}, \ldots, \xi_{n}$. Thus, it remains to invert the cosine transform based on this discrete information to estimate $\varphi$.

This problem has already been addressed in, e.g. [7] and [12]. Here, we focus on the method presented in [10], where it has been shown that the inversion can be done in a numerically stable way with the method of the approximate inverse, introduced in [9]; see also [15]. The idea is to calculate a 'smoothed version' of $\varphi$, denoted by $\varphi_{\gamma}$ for some $\gamma>0$, which is defined as

$$
\varphi_{\gamma}(\eta)=\int_{S^{d-1}} \varphi(\xi) e_{\gamma}(\eta, \xi) \mathrm{d} \xi, \quad \eta \in S^{d-1}
$$

where we assume that $\varphi_{\gamma} \rightarrow \varphi$ in some sense as $\gamma$ tends to 0 . For the so-called mollifier $e_{\gamma} \in L^{2}\left(S^{d-1} \times S^{d-1}\right)$, it is required that

$$
\int_{S^{d-1}} e_{\gamma}(\eta, \xi) \mathrm{d} \xi=1, \quad \eta \in S^{d-1} .
$$

For a given mollifier, the reconstruction kernel $\psi_{\gamma}$ is defined as the solution of $e_{\gamma}=C \psi_{\gamma}$. Since the cosine transform $C$ is self-adjoint, this allows us to write the smoothed density as

$$
\varphi_{\gamma}(\eta)=\left\langle\varphi, e_{\gamma}(\eta, \cdot)\right\rangle_{L^{2}\left(S^{d-1}\right)}=\left\langle\varphi, C \psi_{\gamma}(\eta, \cdot)\right\rangle_{L^{2}\left(S^{d-1}\right)}=\left\langle C \varphi, \psi_{\gamma}(\eta, \cdot)\right\rangle_{L^{2}\left(S^{d-1}\right)},
$$

where the last term is the inner product of two known functions, namely the given data $C \varphi$ and the reconstruction kernel. This also has computational advantages as the reconstruction kernel can be calculated in advance (independent of the given data $C \varphi$ ), and, thus, the approximate inversion for one density requires only the calculation of an inner product. For $d=2$ and $d=3$, explicit formulae of reconstruction kernels for some interesting mollifiers have been derived in [10]. See Corollary 3 in Appendix B for reconstruction kernels of Gaussian mollifiers in arbitrary dimensions $d \geq 3$.

In this paper we make the additional assumption on the mollifier $e_{\gamma}(\eta, \xi)$ (and, thus, also on the reconstruction kernel $\left.\psi_{\gamma}(\eta, \xi)\right)$ that it depends only on the geodesic distance between $\xi$ and $\eta$.

Although this method can be used for arbitrary stationary fiber processes, in this paper we restrict to Poisson line processes (cf. [14, Chapter 4.4]). Our considerations also hold for Poisson cylinder processes, see [17] or [20], if the number of cylinders hitting a test set can be observed. One should remark that similar results can be obtained for other stationary Poisson processes, e.g. processes of line segments.

Throughout this paper, we use the following notation for the intersection counts. We assume that the number of intersections of $\Xi$ with hyperplanes orthogonal to the unit vectors $\xi_{1 n}, \ldots, \xi_{n n} \in S^{d-1}$ in a window $\varrho W=b_{\varrho}(\mathbf{o})$ can be observed. These intersection counts are denoted by $Y_{1 n}, \ldots, Y_{n n}$, i.e. $Y_{i n}=\#\left\{\Xi \cap \xi_{i n}^{\perp} \cap \varrho W\right\}$. To simplify the notation, we will 
write $\xi_{i}$ instead of $\xi_{i n}$ when the value of $n$ is clear, and analogously $Y_{i}$ for $Y_{i n}$. Since $\Xi$ is a stationary Poisson line process, the process $\Xi \cap \xi_{i n}^{\perp}$ is also stationary. It has the intensity $\lambda C \varphi\left(\xi_{\text {in }}\right)$ (see [14, Theorem 4.4.6]), and, thus, $Y_{i n} \sim \operatorname{Poi}\left(\kappa_{d-1} \varrho^{d-1} \lambda C \varphi\left(\xi_{\text {in }}\right)\right)$, where $\kappa_{d-1}$ is the volume of the unit ball in $\mathbb{R}^{d-1}$.

If the intensity $\lambda$ of the Poisson line process is known, we introduce the notation $\tilde{Y}_{i}^{\lambda}=$ $Y_{i} / \kappa_{d-1} \varrho^{d-1} \lambda$, which leads to $\mathrm{E} \tilde{Y}_{i}^{\lambda}=C \varphi\left(\xi_{i}\right)$ and $\operatorname{var} \tilde{Y}_{i}^{\lambda}=C \varphi\left(\xi_{i}\right) / \kappa_{d-1} \varrho^{d-1} \lambda$ for all $\varrho, n$, and $i$.

Now, we can introduce the estimator

$$
\hat{\varphi}_{\gamma}^{\lambda}(\eta):=\sum_{i=1}^{n} \tilde{Y}_{i}^{\lambda} \psi_{\gamma}\left(\eta, \xi_{i}\right) \Delta_{i}
$$

for the density $\varphi$ at the point $\eta \in S^{d-1}$, which can be seen as a discretized version of (2). Here, the weight $\Delta_{i}$ is defined as follows. Introduce the spherical Voronoi cell at $\xi_{i}$ as

$$
c\left(\xi_{i}\right)=\left\{v \in S^{d-1}: d\left(v, \xi_{i}\right) \leq \min \left\{d\left(v, \xi_{j}\right), d\left(v,-\xi_{j}\right)\right\} \text { for all } j \neq i\right\},
$$

where $d(\cdot, \cdot)$ denotes the geodesic distance, i.e. the length of the shortest path in $S^{d-1}$ between two unit vectors. Then we set the weight $\Delta_{i}$ of the estimator (3) to be two times the area of the Voronoi cell $c\left(\xi_{i}\right)$ at $\xi_{i}$, which leads to $\sum_{i=1}^{n} \Delta_{i}=\omega_{d-1}$, where $\omega_{d-1}$ is the surface area of $S^{d-1}$.

If $\lambda$ is unknown, we use the following estimator for the intensity:

$$
\hat{\lambda}:=\frac{1}{2 \kappa_{d-1}} \sum_{i=1}^{n} \frac{Y_{i}}{\kappa_{d-1} \varrho^{d-1}} \Delta_{i} .
$$

Furthermore, we introduce the notation $\tilde{Y}_{i}=Y_{i} / \kappa_{d-1} \varrho^{d-1} \hat{\lambda}$ analogously to the case of known intensity, where we use the convention $0 / 0=0$ in the case $\hat{\lambda}=0$, i.e. $Y_{i}=0$ for $i=1, \ldots, n$. Here, we use the estimator

$$
\hat{\varphi}_{\gamma}(\eta):=\sum_{i=1}^{n} \tilde{Y}_{i} \psi_{\gamma}\left(\eta, \xi_{i}\right) \Delta_{i}=\frac{\lambda}{\hat{\lambda}} \hat{\varphi}_{\gamma}^{\lambda}(\eta) .
$$

Remark 1. The formulae for the estimators in (3) and (4) have a form similar to kernel density estimators (KDEs). However, here, one has to solve an inverse problem instead of observing the searched-for random variable directly (or with some additional noise). As a result, the reconstruction kernel $\psi_{\gamma}$ has a different form to the usual KDE kernels (see Figure 1) and the estimators cannot be regarded as standard KDEs.

Note that the supremum of the absolute value of the reconstruction kernel $\psi_{\gamma}$ tends to $\infty$ for $\gamma \rightarrow 0$. Therefore, $\hat{\varphi}_{\gamma}^{\lambda}$ (and $\hat{\varphi}_{\gamma}$ ) does not converge to $\varphi$ pointwise or in the $L^{2}\left(S^{d-1}\right)$-sense. To overcome this, we fix $\gamma>0$ and analyze the properties of the estimator $\hat{\varphi}_{\gamma}^{\lambda}$, especially the convergence to the mollified density $\varphi_{\gamma}$ as $\varrho, n \rightarrow \infty$. For suitable mollifiers (cf. [10]), $\varphi_{\gamma}$ approximates $\varphi$ as $\gamma \rightarrow 0$.

This paper is organized as follows. In the next section, strong convergence of the estimators $\hat{\varphi}_{\gamma}^{\lambda}$ and $\hat{\varphi}_{\gamma}$ to the mollified density $\varphi_{\gamma}$ is analyzed. We show that the supremum of the difference between the two functions converges almost surely to 0 under mild assumptions on the directions of the test hyperplanes and the growth rate of the observation window radius. In Section 3, Berry-Esseen bounds for the estimator $\hat{\varphi}_{\gamma}^{\lambda}$ are derived, and a central limit theorem is used to construct asymptotic tests on the directional distribution. In Section 4, the large deviation behavior of $\hat{\varphi}_{\gamma}^{\lambda}$ is studied and in the final section, some results of simulation studies are presented. 

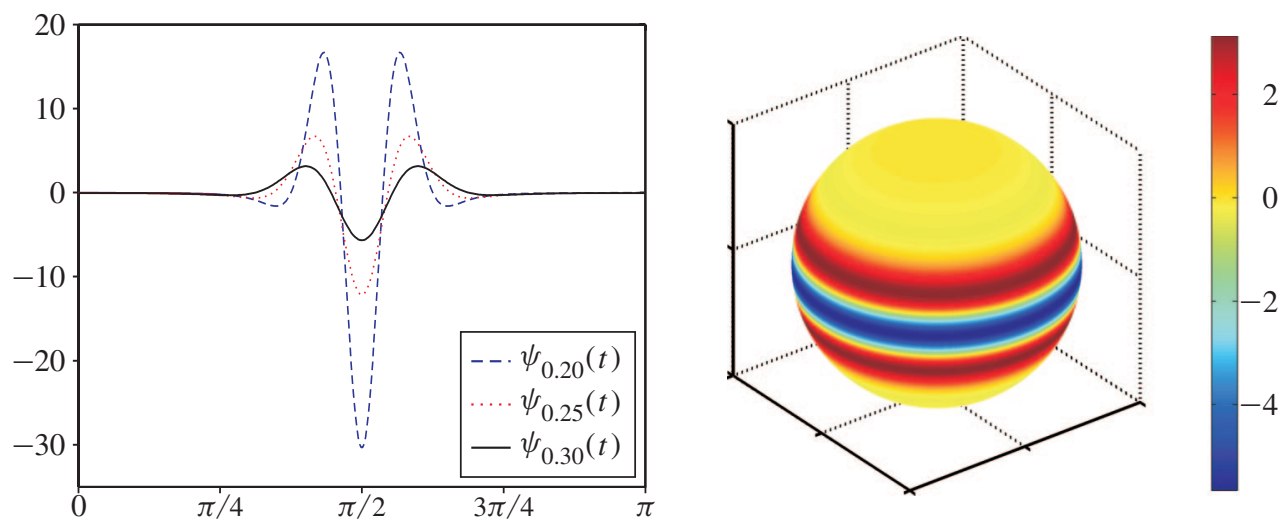

FIgURE 1: The Gaussian reconstruction kernel in three dimensions as a function of the polar angle and its graph on the sphere (with the north pole the reconstruction point).

\section{Almost-sure convergence}

We derive sufficient conditions for almost-sure convergence of the estimator $\hat{\varphi}_{\gamma}^{\lambda}$ in the supremum norm, i.e.

$$
\sup _{\eta \in S^{d-1}}\left|\hat{\varphi}_{\gamma}^{\lambda}(\eta)-\varphi_{\gamma}(\eta)\right| \rightarrow 0 \quad \text { as } n \rightarrow \infty
$$

For this, some results on the convergence speed of the estimator are derived (in Lemma 3 below), which are of interest in their own right. Yet, we focus on the almost-sure convergence, so no attempt has been made to achieve optimality in the convergence rates.

In this section, a frequent prerequisite is that the reconstruction kernel is Lipschitz continuous. By this, we mean that it is Lipschitz continuous in both components, i.e. for $\eta, \xi_{1}, \xi_{2} \in S^{d-1}$, we have

$$
\left|\psi_{\gamma}\left(\eta, \xi_{1}\right)-\psi_{\gamma}\left(\eta, \xi_{2}\right)\right| \leq L_{\psi_{\gamma}} d\left(\xi_{1}, \xi_{2}\right)
$$

for some $L_{\psi_{\gamma}}<\infty$, and the same holds for the first component if we fix the second. Since $S^{d-1}$ is compact, this also means that $\psi_{\gamma}$ is bounded.

Similarly to [4], we define the symmetrized mesh norm of a set of points $\left\{\xi_{1}, \ldots, \xi_{n}\right\}$ as the mesh norm of the set $\left\{\xi_{1}, \ldots, \xi_{n},-\xi_{1}, \ldots,-\xi_{n}\right\}$, i.e.

$$
h^{*}\left(\xi_{1}, \ldots, \xi_{n}\right)=h\left(\xi_{1}, \ldots, \xi_{n},-\xi_{1}, \ldots,-\xi_{n}\right)=\max _{v \in S^{d-1}} \min _{1 \leq i \leq n}\left\{d\left(v, \xi_{i}\right), d\left(v,-\xi_{i}\right)\right\}
$$

Note that the weights $\Delta_{i}, i=1, \ldots, n$, of the estimator (3) are thus bounded by $2 \kappa_{d-1} h^{*}\left(\xi_{1}\right.$, $\left.\ldots, \xi_{n}\right)^{d-1}$, as the maximal geodesic radius of one cell is $h^{*}\left(\xi_{1}, \ldots, \xi_{n}\right)$. Throughout this section, we use the following auxiliary result on the cosine transform.

Lemma 1. For arbitrary densities $\varphi$ on $S^{d-1}$, the cosine transform $C \varphi$ is Lipschitz continuous with respect to the geodesic distance. The Lipschitz constant is at most 1 . Furthermore, $C \varphi$ is bounded by 1 . 
Proof. For $\zeta, \eta \in S^{d-1}$, we have

$$
\begin{aligned}
|C \varphi(\zeta)-C \varphi(\eta)| & =\left|\int_{S^{d-1}}(|\langle\zeta, v\rangle|-|\langle\eta, v\rangle|) \varphi(v) \mathrm{d} v\right| \\
& \leq \sup _{v \in S^{d-1}}||\langle\zeta, v\rangle|-|\langle\eta, v\rangle|| \\
& \leq d(\zeta, \eta) .
\end{aligned}
$$

The inequality $C \varphi(\zeta)=\int_{S^{d-1}}|\langle\zeta, v\rangle| \varphi(\nu) \mathrm{d} \nu \leq 1$ completes the proof.

\subsection{Deterministic measurement directions}

At first we assume that the series of measurement directions is deterministic. See, e.g. [5] or [16] for an approach to choose a suitable point configuration.

Let us begin with the analysis of the bias of the estimator (3). Note that $\mathrm{E} \tilde{Y}_{i n}^{\lambda}=C \varphi\left(\xi_{i n}\right)$ does not depend on the observation window radius $\varrho$.

Theorem 1. Let the reconstruction kernel $\psi_{\gamma}$ be Lipschitz continuous, and denote by $L_{C \varphi}$ and $L_{\psi_{\gamma}}$ the Lipschitz constants of C $\varphi$ and $\psi_{\gamma}$, respectively. Then

$$
\sup _{\eta \in S^{d-1}}\left|\mathrm{E} \hat{\varphi}_{\gamma}^{\lambda}(\eta)-\varphi_{\gamma}(\eta)\right| \leq \omega_{d-1}\left(L_{C \varphi} \bar{\psi}_{\gamma}+L_{\psi_{\gamma}}\right) h^{*}\left(\xi_{1}, \ldots, \xi_{n}\right)
$$

uniformly for all $\varrho>0$, where $\bar{\psi}_{\gamma}$ denotes the supremum of $\left|\psi_{\gamma}\right|$.

Proof. Let $\eta \in S^{d-1}$ be an arbitrary unit vector. Then, for $v \in c\left(\xi_{i}\right), i=1, \ldots, n$,

$$
\begin{aligned}
\mid C \varphi\left(\xi_{i}\right) & \psi_{\gamma}\left(\eta, \xi_{i}\right)-C \varphi(v) \psi_{\gamma}(\eta, v) \mid \\
& \leq\left|C \varphi\left(\xi_{i}\right) \psi_{\gamma}\left(\eta, \xi_{i}\right)-C \varphi\left(\xi_{i}\right) \psi_{\gamma}(\eta, v)\right|+\left|C \varphi\left(\xi_{i}\right) \psi_{\gamma}(\eta, v)-C \varphi(v) \psi_{\gamma}(\eta, v)\right| \\
& =\left|C \varphi\left(\xi_{i}\right)\right|\left|\psi_{\gamma}\left(\eta, \xi_{i}\right)-\psi_{\gamma}(\eta, v)\right|+\left|\psi_{\gamma}(\eta, v)\right|\left|C \varphi\left(\xi_{i}\right)-C \varphi(v)\right| \\
& \leq L_{\psi_{\gamma}} h^{*}\left(\xi_{1}, \ldots, \xi_{n}\right)+\bar{\psi}_{\gamma} L_{C \varphi} h^{*}\left(\xi_{1}, \ldots, \xi_{n}\right),
\end{aligned}
$$

since $C \varphi$ is bounded by 1 (see Lemma 1). Because $C \varphi$ and $\psi_{\gamma}$ are symmetric functions, we finally obtain

$$
\begin{aligned}
\left|\mathrm{E} \hat{\varphi}_{\gamma}^{\lambda}(\eta)-\varphi_{\gamma}(\eta)\right| & =\left|\sum_{i=1}^{n} C \varphi\left(\xi_{i}\right) \psi_{\gamma}\left(\eta, \xi_{i}\right) \Delta_{i}-\int_{S^{d-1}} C \varphi(v) \psi_{\gamma}(\eta, v) \mathrm{d} v\right| \\
& \leq 2 \sum_{i=1}^{n} \int_{c\left(\xi_{i}\right)}\left|C \varphi\left(\xi_{i}\right) \psi_{\gamma}\left(\eta, \xi_{i}\right)-C \varphi(v) \psi_{\gamma}(\eta, v)\right| \mathrm{d} v \\
& \leq \omega_{d-1}\left(L_{C \varphi} \bar{\psi}_{\gamma}+L_{\psi_{\gamma}}\right) h^{*}\left(\xi_{1}, \ldots, \xi_{n}\right) .
\end{aligned}
$$

To analyze the weak convergence of $\hat{\varphi}_{\gamma}^{\lambda}$, we need the following lemma.

Lemma 2. If $\left|\psi_{\gamma}\right|$ is bounded by $\bar{\psi}_{\gamma}<\infty$ then, for $\varepsilon>0$,

$$
\mathrm{P}\left(\sup _{\eta \in S^{d-1}} \sum_{i=1}^{n}\left|\left(\tilde{Y}_{i}^{\lambda}-C \varphi\left(\xi_{i}\right)\right) \psi_{\gamma}\left(\eta, \xi_{i}\right)\right| \Delta_{i}>\varepsilon\right) \leq \frac{\omega_{d-1}^{2} \bar{\psi}_{\gamma}^{2}}{\kappa_{d-1} \varrho^{d-1} \lambda \varepsilon^{2}}
$$

uniformly for all $n \in \mathbb{N}$. 
Proof. Using the notation $Y(\xi)=\#\left\{\Xi \cap \xi^{\perp} \cap \varrho W\right\}$ and $\tilde{Y}^{\lambda}(\xi)=Y(\xi) / \kappa_{d-1} \varrho^{d-1} \lambda$, we obtain

$$
Y(\xi) \sim \operatorname{Poi}\left(\kappa_{d-1} \varrho^{d-1} \lambda C \varphi(\xi)\right) \quad \text { and } \quad \operatorname{var} \tilde{Y}^{\lambda}(\xi)=\frac{C \varphi(\xi)}{\kappa_{d-1} \varrho^{d-1} \lambda} .
$$

Lemma 1 and Chebyshev's inequality lead to

$$
\begin{aligned}
\mathrm{P}\left(\sup _{\eta \in S^{d-1}} \sum_{i=1}^{n}\left|\left(\tilde{Y}_{i}^{\lambda}-C \varphi\left(\xi_{i}\right)\right) \psi_{\gamma}\left(\eta, \xi_{i}\right)\right| \Delta_{i}>\varepsilon\right) & \leq \mathrm{P}\left(\sum_{i=1}^{n}\left|\tilde{Y}_{i}^{\lambda}-C \varphi\left(\xi_{i}\right)\right| \Delta_{i}>\frac{\varepsilon}{\bar{\psi}_{\gamma}}\right) \\
& \leq \frac{\bar{\psi}_{\gamma}^{2}}{\varepsilon^{2}} \operatorname{var}\left(\sum_{i=1}^{n}\left|\tilde{Y}_{i}^{\lambda}-C \varphi\left(\xi_{i}\right)\right| \Delta_{i}\right) \\
& \leq \frac{\omega_{d-1}^{2} \bar{\psi}_{\gamma}^{2}}{\varepsilon^{2}} \sup _{\nu \in S^{d-1}} \operatorname{var}\left(\left|\tilde{Y}^{\lambda}(\nu)-C \varphi(v)\right|\right) \\
& \leq \frac{\omega_{d-1}^{2} \bar{\psi}_{\gamma}^{2}}{\varepsilon^{2}} \sup _{\nu \in S^{d-1}} \frac{C \varphi(\nu)}{\kappa_{d-1} \varrho^{d-1} \lambda} \\
& \leq \frac{\omega_{d-1}^{2} \bar{\psi}_{\gamma}^{2}}{\kappa_{d-1} \varrho^{d-1} \lambda \varepsilon^{2}} .
\end{aligned}
$$

Lemma 3. Let $\psi_{\gamma}$ be Lipschitz continuous. If $h^{*}\left(\xi_{1}, \ldots, \xi_{n}\right)<c_{h}^{-1} \varepsilon$,

$$
\mathrm{P}\left(\sup _{\eta \in S^{d-1}}\left|\hat{\varphi}_{\gamma}^{\lambda}(\eta)-\varphi_{\gamma}(\eta)\right|>\varepsilon\right) \leq \frac{\omega_{d-1}^{2} \bar{\psi}_{\gamma}^{2}}{\kappa_{d-1} \varrho^{d-1} \lambda\left(\varepsilon-c_{h} h^{*}\left(\xi_{1}, \ldots, \xi_{n}\right)\right)^{2}},
$$

where the constant $c_{h}=\omega_{d-1}\left(L_{C \varphi} \bar{\psi}_{\gamma}+L_{\psi_{\gamma}}\right)$ does not depend on $\xi_{1}, \ldots, \xi_{n}$.

Proof. The main idea of the proof is to split our estimator into two parts:

$$
\hat{\varphi}_{\gamma}^{\lambda}(\eta)=\sum_{i=1}^{n}\left(\tilde{Y}_{i}^{\lambda}-C \varphi\left(\xi_{i}\right)\right) \psi_{\gamma}\left(\eta, \xi_{i}\right) \Delta_{i}+\sum_{i=1}^{n} C \varphi\left(\xi_{i}\right) \psi_{\gamma}\left(\eta, \xi_{i}\right) \Delta_{i} .
$$

For the specified $h^{*}$, Lemma 2 and the proof of Theorem 1 lead to

$$
\begin{aligned}
\mathrm{P}\left(\sup _{\eta \in S^{d-1}}\left|\hat{\varphi}_{\gamma}^{\lambda}(\eta)-\varphi_{\gamma}(\eta)\right|>\varepsilon\right) \leq \mathrm{P}\left(\operatorname { s u p } _ { \eta \in S ^ { d - 1 } } \left(\sum_{i=1}^{n}\left|\left(\tilde{Y}_{i}^{\lambda}-C \varphi\left(\xi_{i}\right)\right) \psi_{\gamma}\left(\eta, \xi_{i}\right)\right| \Delta_{i}\right.\right. \\
\left.\left.+\left|\sum_{i=1}^{n} C \varphi\left(\xi_{i}\right) \psi_{\gamma}\left(\eta, \xi_{i}\right) \Delta_{i}-\varphi_{\gamma}(\eta)\right|\right)>\varepsilon\right) \\
\leq \mathrm{P}\left(\sup _{\eta \in S^{d-1}} \sum_{i=1}^{n}\left|\left(\tilde{Y}_{i}^{\lambda}-C \varphi\left(\xi_{i}\right)\right) \psi_{\gamma}\left(\eta, \xi_{i}\right)\right| \Delta_{i}>\varepsilon\right. \\
\left.-\omega_{d-1}\left(L_{C \varphi} \bar{\psi}_{\gamma}+L_{\psi_{\gamma}}\right) h^{*}\left(\xi_{1}, \ldots, \xi_{n}\right)\right) \\
\leq \frac{\omega_{d-1}^{2} \bar{\psi}_{\gamma}^{2}}{\kappa_{d-1} \varrho^{d-1} \lambda\left(\varepsilon-c_{h} h^{*}\left(\xi_{1}, \ldots, \xi_{n}\right)\right)^{2}} .
\end{aligned}
$$


This immediately leads to the following result.

Theorem 2. Suppose that $\psi_{\gamma}$ is Lipschitz continuous, the symmetrized mesh norm $h^{*}\left(\xi_{1 n}, \ldots\right.$, $\left.\xi_{n n}\right)$ tends to 0 , and $\varrho=\varrho(n) \geq c_{1} n^{\left(1+c_{2}\right) /(d-1)}$ for some constants $c_{1}, c_{2}>0$. Then, almost surely,

$$
\sup _{\eta \in S^{d-1}}\left|\hat{\varphi}_{\gamma}^{\lambda}(\eta)-\varphi_{\gamma}(\eta)\right| \rightarrow 0 \quad \text { as } n \rightarrow \infty
$$

Proof. The result follows from the Borel-Cantelli lemma. Since $h^{*}\left(\xi_{1 n}, \ldots, \xi_{n n}\right)$ tends to 0 , there exists an integer $N$ such that $h^{*}\left(\xi_{1 n}, \ldots, \xi_{n n}\right) \leq \frac{1}{2} c_{h}^{-1} \varepsilon$ for $n \geq N$. Thus, with Lemma 3 we obtain, for arbitrary $\varepsilon>0$,

$$
\sum_{n=1}^{\infty} \mathrm{P}\left(\sup _{\eta \in S^{d-1}}\left|\hat{\varphi}_{\gamma}^{\lambda}(\eta)-\varphi_{\gamma}(\eta)\right|>\varepsilon\right) \leq N+\sum_{n=N+1}^{\infty} \frac{4 \omega_{d-1}^{2} \bar{\psi}_{\gamma}^{2}}{\kappa_{d-1}\left(c_{1} n^{\left(1+c_{2}\right) /(d-1)}\right)^{d-1} \lambda \varepsilon^{2}}<\infty .
$$

\subsection{Random measurement directions}

In this section, instead of deterministic measurement directions, we consider the following setting which should be a suitable model for applications in which the measurement directions cannot be chosen but are given in a random way. We consider as measurement directions the series of independent and identically distributed (i.i.d.) unit vectors $\xi_{1}, \xi_{2}, \ldots$, which are assumed to be independent of $\Xi$. For the estimator (3), we use the same weights as in the deterministic setting. See also Remark 2 below for a note on other point configurations.

Lemma 4. Let $\xi_{1}, \xi_{2}, \ldots$ be a sequence of i.i.d. random unit vectors with positive density with respect to the spherical surface measure. Then $h^{*} \rightarrow 0$ almost surely.

Proof. To simplify the notation, we show the stronger claim that the nonsymmetrized mesh norm $h\left(\xi_{1}, \ldots, \xi_{n}\right)$ tends to 0 almost surely. Let $\varepsilon>0$. Obviously, there exists some integer $m(\varepsilon)<\infty$ and a sequence of unit vectors $v_{1}, \ldots, v_{m} \in S^{d-1}$ with $h\left(v_{1}, \ldots, v_{m}\right)<\varepsilon / 2$. As the density of $\xi_{1}$ is positive, for every cell $c\left(v_{i}\right)$ of the tessellation induced by $v_{1}, \ldots, v_{m}$, it holds that $\mathrm{P}\left(\xi_{1} \in c\left(v_{i}\right)\right)>0$. Thus, there is a constant $p$ with $0<p \leq \mathrm{P}\left(\xi_{1} \in c\left(v_{i}\right)\right), i=1, \ldots, m$. With this, the probability that there is a cell which contains none of the vectors $\xi_{1}, \ldots, \xi_{n}$ can be estimated from above by

$\mathrm{P}\left(\right.$ there exists $\left.i:\left\{\xi_{1}, \ldots, \xi_{n}\right\} \cap c\left(v_{i}\right)=\varnothing\right) \leq \sum_{i=1}^{m} \mathrm{P}\left(\left\{\xi_{1}, \ldots, \xi_{n}\right\} \cap c\left(v_{i}\right)=\varnothing\right) \leq m(1-p)^{n}$.

Since $\sum_{n=1}^{\infty} m(1-p)^{n}<\infty$, it follows from the Borel-Cantelli lemma that every cell contains at least one point almost surely as $n$ tends to $\infty$. Thus, the mesh norm of the sequence $\xi_{1}, \ldots, \xi_{n}$ can at most be $\varepsilon$.

Theorem 3. Let $\xi_{1}, \xi_{2}, \ldots$ be a sequence of i.i.d. random unit vectors with nonnegative density with respect to the spherical surface measure. Then

$$
\sup _{\eta \in S^{d-1}}\left|\mathrm{E} \hat{\varphi}_{\gamma}^{\lambda}(\eta)-\varphi_{\gamma}(\eta)\right| \rightarrow 0 \text { as } n \rightarrow \infty \text {. }
$$

If $\psi_{\gamma}$ is Lipschitz continuous and the radii fulfill $\varrho(n) \geq c_{1} n^{\left(1+c_{2}\right) /(d-1)}$ for some $c_{1}, c_{2}>0$,

$$
\sup _{\eta \in S^{d-1}}\left|\hat{\varphi}_{\gamma}^{\lambda}(\eta)-\varphi_{\gamma}(\eta)\right| \rightarrow 0 \quad \text { as } n \rightarrow \infty
$$

holds almost surely. 
Proof. This is a direct consequence of Lemma 4 in combination with Theorem 1 and Theorem 2, respectively.

Remark 2. The same holds for any other sequence of random points whose symmetrized mesh norm tends to 0 , e.g. in the case of systematic sampling.

\subsection{Unknown intensity}

In this section we analyze the estimator $\hat{\varphi}_{\gamma}$, defined in (4), which can be used if $\lambda$ is unknown. We begin with the following auxiliary result.

Lemma 5. Let $\Xi$ be a stationary Poisson line process with directional distribution density $\varphi$ and intensity $\lambda>0$. If the symmetrized mesh norm $h^{*}\left(\xi_{1 n}, \ldots, \xi_{n n}\right)$ of the measurement directions tends to 0 , and $\varrho(n) \geq c_{1} n^{\left(1+c_{2}\right) /(d-1)}$ for some constants $c_{1}, c_{2}>0$, then the estimator $\hat{\lambda}$ for the intensity of $\Xi$ is strongly consistent for $n \rightarrow \infty$.

Proof. For $\varepsilon>0$, we have

$$
\begin{aligned}
\mathrm{P}(|\hat{\lambda}-\lambda|>\varepsilon)= & \mathrm{P}\left(\left|\frac{1}{2 \kappa_{d-1}} \sum_{i=1}^{n} \frac{Y_{i}}{\kappa_{d-1} \varrho^{d-1}} \Delta_{i}-\lambda\right|>\varepsilon\right) \\
\leq & \mathrm{P}\left(\left|\frac{1}{2 \kappa_{d-1}} \sum_{i=1}^{n} \frac{Y_{i}-\mathrm{E} Y_{i}}{\kappa_{d-1} \varrho^{d-1}} \Delta_{i}\right|>\frac{\varepsilon}{2}\right) \\
& +\mathrm{P}\left(\left|\frac{1}{2 \kappa_{d-1}} \sum_{i=1}^{n} \frac{\mathrm{E} Y_{i}}{\kappa_{d-1} \varrho^{d-1}} \Delta_{i}-\lambda\right|>\frac{\varepsilon}{2}\right) .
\end{aligned}
$$

For the first probability in (5), repeating the steps in the proof of Lemma 2 leads to

$$
\mathrm{P}\left(\left|\frac{1}{2 \kappa_{d-1}} \sum_{i=1}^{n} \frac{Y_{i}-\mathrm{E} Y_{i}}{\kappa_{d-1} \varrho^{d-1}} \Delta_{i}\right|>\frac{\varepsilon}{2}\right) \leq \frac{\omega_{d-1}^{2} \lambda}{\kappa_{d-1}^{3} \varrho^{d-1} \varepsilon^{2}}
$$

For the second summand in (5), it follows from [3, p. 428] that

$$
\int_{S^{d-1}} C \varphi(\xi) \mathrm{d} \xi=\int_{S^{d-1}} \int_{S^{d-1}}|\langle\xi, \nu\rangle| \varphi(\nu) \mathrm{d} \nu \mathrm{d} \xi=\int_{S^{d-1}} \int_{S^{d-1}}|\langle\xi, \nu\rangle| \mathrm{d} \xi \varphi(\nu) \mathrm{d} \nu=2 \kappa_{d-1}
$$

The Lipschitz continuity of $C \varphi$ leads to

$$
\begin{aligned}
\left|\sum_{i=1}^{n} C \varphi\left(\xi_{i}\right) \Delta_{i}-\int_{S^{d-1}} C \varphi(\xi) \mathrm{d} \xi\right| & \leq 2 \sum_{i=1}^{n} \int_{c\left(\xi_{i}\right)}\left|C \varphi\left(\xi_{i}\right)-C \varphi(\xi)\right| \mathrm{d} \xi \\
& \leq \omega_{d-1} h^{*}\left(\xi_{1 n}, \ldots, \xi_{n n}\right) \\
& \rightarrow 0 \text { as } n \rightarrow \infty,
\end{aligned}
$$

since $h^{*}\left(\xi_{1 n}, \ldots, \xi_{n n}\right)$ tends to 0 as $n$ tends to $\infty$. Thus, the second term in (5) is 0 for large enough $n$.

Combined, this means that the sum $\sum_{n=1}^{\infty} \mathrm{P}(|\hat{\lambda}(n)-\lambda|>\varepsilon)$ is finite if $\sum_{n=1}^{\infty} 1 / \varrho^{d-1}$ is. This is clearly the case under the condition on $\varrho(n)$ above. The claim follows from the BorelCantelli lemma.

Now, we are in a position to formulate the main result for this section. 
Theorem 4. In the case of deterministic measurement directions, under the assumptions of Theorem 2, almost surely,

$$
\sup _{\eta \in S^{d-1}}\left|\hat{\varphi}_{\gamma}(\eta)-\varphi_{\gamma}(\eta)\right| \rightarrow 0 \quad \text { as } n \rightarrow \infty .
$$

Furthermore, in the case of random measurement directions, if the conditions of the second claim in Theorem 3 hold, almost surely,

$$
\sup _{\eta \in S^{d-1}}\left|\hat{\varphi}_{\gamma}(\eta)-\varphi_{\gamma}(\eta)\right| \rightarrow 0 \text { as } n \rightarrow \infty
$$

Proof. This is an immediate consequence of the respective theorems, Lemma 5, and the fact that $\hat{\varphi}_{\gamma}(\eta)=(\lambda / \hat{\lambda}) \hat{\varphi}_{\gamma}^{\lambda}(\eta)$.

Remark 3. Again, Theorem 4 also holds for any other sequence of random points whose symmetrized mesh norm tends to 0 .

\section{Berry-Esseen bounds}

In this section we show how, for some fixed $\eta \in S^{d-1}$, the estimator $\hat{\varphi}_{\gamma}^{\lambda}(\eta)$ can be written as a compound Poisson process and we use some well-known results to derive Berry-Esseen bounds; see [8]. Since we are interested in the distribution of $\hat{\varphi}_{\gamma}^{\lambda}$, we can simplify the notation in the following way. The line process is stationary, so instead of increasing the radius of the observation window, we can also increase the intensity, which will have the same effect on the estimator. Thus, it suffices to let $\lambda$ tend to $\infty$ and restrict to $\varrho=1$, i.e. $\varrho W=W$. We begin with some notation. Denote by $N$ the number of observed lines, i.e. lines which hit $W$. Then $N \sim \operatorname{Poi}\left(\kappa_{d-1} \lambda\right)$. Introducing the symbol $Q$ for the distribution of a typical line of $\Xi$ hitting $W$, we find that $\Xi$ is distributed as the set of the i.i.d. lines $L_{1}, \ldots, L_{N}$ with distribution $Q$, which are independent of $N$.

The definition of the estimator

$$
\hat{\varphi}_{\gamma}^{\lambda}(\eta)=\sum_{i=1}^{n} \tilde{Y}_{i}^{\lambda} \psi_{\gamma}\left(\eta, \xi_{i}\right) \Delta_{i}
$$

can be rewritten as follows. For a line $l$, let $\hat{\varphi}_{\gamma}^{\lambda}(l, \eta)$ be the estimator for the process when $\Xi=\{l\}$. Analogously, define

$$
\tilde{Y}_{i}^{\lambda}(l)=\frac{1}{\kappa_{d-1} \lambda} \mathbf{1}\left\{l \cap \xi_{i} \cap W \neq \varnothing\right\} .
$$

This leads to

$$
\hat{\varphi}_{\gamma}^{\lambda}(\eta)=\sum_{l \in \Xi} \hat{\varphi}_{\gamma}^{\lambda}(l, \eta)=\sum_{l \in \Xi} \sum_{i=1}^{n} \tilde{Y}_{i}^{\lambda}(l) \psi_{\gamma}\left(\eta, \xi_{i}\right) \Delta_{i} \stackrel{\mathrm{D}}{=} \sum_{j=1}^{N} \sum_{i=1}^{n} \tilde{Y}_{i}^{\lambda}\left(L_{j}\right) \psi_{\gamma}\left(\eta, \xi_{i}\right) \Delta_{i} .
$$

As the terms $\sum_{i=1}^{n} \tilde{Y}_{i}^{\lambda}\left(L_{j}\right) \psi_{\gamma}\left(\eta, \xi_{i}\right) \Delta_{i}, j=1,2, \ldots$, are i.i.d. random variables, this means that $\hat{\varphi}_{\gamma}^{\lambda}(\eta)$ has a compound Poisson distribution with size $N$ and summands $\sum_{i=1}^{n} \tilde{Y}_{i}^{\lambda}\left(L_{0}\right) \times$ $\psi_{\gamma}\left(\eta, \xi_{i}\right) \Delta_{i}$, where $L_{0} \sim Q$ is a typical line of $\Xi$ hitting $W$. Thus, we can apply the following well-known result, where we use the constant for the upper bound from [8]. 
Lemma 6. Let $S_{N}=X_{1}+\cdots+X_{N}$ be a compound Poisson process, where $X_{1}, X_{2}, \ldots$ are uniformly distributed with $\mathrm{E}\left|X_{1}\right|^{3}<\infty, N \sim \operatorname{Poi}(\mu)$, and $N, X_{1}, X_{2}, \ldots$ are independent. Then

$$
\sup _{x}\left|\mathrm{P}\left(\frac{S_{N}-\mu \mathrm{E} X_{1}}{\sqrt{\mu \mathrm{E}\left|X_{1}\right|^{2}}} \leq x\right)-\Phi(x)\right| \leq 0.3041 \frac{\mathrm{E}\left|X_{1}\right|^{3}}{\left(\mathrm{E}\left|X_{1}\right|^{2}\right)^{3 / 2} \sqrt{\mu}} .
$$

Together with the considerations above, this can be used to arrive at the main result of this section.

Theorem 5. Let $\eta \in S^{d-1}$, let $L_{0}$ be a typical line of $\Xi$ hitting $W$, and let $\hat{\varphi}_{\gamma}^{\lambda}\left(L_{0}, \eta\right)$ be as above. If $\left|\psi_{\gamma}\right|$ is bounded then

$$
\sup _{x}\left|F_{\lambda}(x)-\Phi(x)\right| \leq 0.3041 \frac{\mathrm{E}\left(\kappa_{d-1} \lambda\left|\hat{\varphi}_{\gamma}^{\lambda}\left(L_{0}, \eta\right)\right|\right)^{3}}{\left(\mathrm{E}\left(\kappa_{d-1} \lambda \hat{\varphi}_{\gamma}^{\lambda}\left(L_{0}, \eta\right)\right)^{2}\right)^{3 / 2} \sqrt{\kappa_{d-1} \lambda}},
$$

where

$$
F_{\lambda}(x)=\mathrm{P}\left(\frac{\hat{\varphi}_{\gamma}^{\lambda}(\eta)-\kappa_{d-1} \lambda \mathrm{E} \hat{\varphi}_{\gamma}^{\lambda}\left(L_{0}, \eta\right)}{\sqrt{\kappa_{d-1} \lambda \mathrm{E} \hat{\varphi}_{\gamma}^{\lambda}\left(L_{0}, \eta\right)^{2}}} \leq x\right) .
$$

Proof. Since the reconstruction kernel $\psi_{\gamma}$ is bounded, for the third moment, we have $\mathrm{E}\left(\kappa_{d-1} \lambda\left|\hat{\varphi}_{\gamma}^{\lambda}\left(L_{0}, \eta\right)\right|\right)^{3} \leq \omega_{d-1}^{3} \bar{\psi}_{\gamma}^{3}<\infty$; see Theorem 6 below. Thus, Lemma 6 can be applied with $\mu=\lambda \kappa_{d-1}$.

One should remark that on the right-hand side of (6) we have expanded the fraction by $\kappa_{d-1}^{3} \lambda^{3}$ because then the expectation values do not depend on $\lambda$ (and $\kappa_{d-1}$ ). With Theorem 5, it remains to derive (bounds for) the second and third absolute moments of $\sum_{i=1}^{n} \tilde{Y}_{i}^{\lambda}\left(L_{0}\right) \psi_{\gamma}\left(\eta, \xi_{i}\right) \Delta_{i}$. This in particular makes it necessary to determine the mixed moments of $Y_{1}, \ldots, Y_{n}$, i.e. the probability that the typical line hits a set of test hyperplanes within the observation window. For notational ease, we assume that these are the first $m$ of our test hyperplanes, i.e. $\xi_{1}^{\perp}, \ldots, \xi_{m}^{\perp}$ for $m=1,2,3$. Yet, to avoid cumbersome formulae, we restrict to $m=1,2$ and use an upper bound for the third moment. For this calculation, we introduce the notation

$$
H_{1, \ldots, m}=H_{\xi_{1}, \ldots, \xi_{m}}=\left\{l \in \Lambda_{d}: l \cap \xi_{1}^{\perp} \cap W \neq \varnothing, \ldots, l \cap \xi_{m}^{\perp} \cap W \neq \varnothing\right\},
$$

where $\Lambda_{d}$ is the set of all lines in $\mathbb{R}^{d}$. Here $H_{1, \ldots, m}$ is the set of all lines which hit the hyperplanes $\xi_{1}^{\perp}, \ldots, \xi_{m}^{\perp}$ within $W$, which means that

$$
Q\left(H_{1, \ldots, m}\right)=\mathrm{P}\left(L_{0} \cap \xi_{1}^{\perp} \cap W \neq \varnothing, \ldots, L_{0} \cap \xi_{m}^{\perp} \cap W \neq \varnothing\right)=\operatorname{E} Y_{1}\left(L_{0}\right) \cdots Y_{m}\left(L_{0}\right) .
$$

Theorem 6. Let the conditions of Theorem 5 hold. If the measurement directions $\xi_{1}, \ldots, \xi_{n}$ are deterministic then

$$
\begin{gathered}
\mathrm{E}\left(\kappa_{d-1} \lambda \hat{\varphi}_{\gamma}^{\lambda}\left(L_{0}, \eta\right)\right)=\sum_{i=1}^{n} C \varphi\left(\xi_{i}\right) \psi_{\gamma}\left(\eta, \xi_{i}\right) \Delta_{i} \\
\mathrm{E}\left(\kappa_{d-1} \lambda \hat{\varphi}_{\gamma}^{\lambda}\left(L_{0}, \eta\right)\right)^{2}=\sum_{i=1}^{n} \sum_{j=1}^{n} \psi_{\gamma}\left(\eta, \xi_{i}\right) \psi_{\gamma}\left(\eta, \xi_{j}\right) \Delta_{i} \Delta_{j} Q\left(H_{i, j}\right)
\end{gathered}
$$

do not depend on $\lambda$.

For any (possibly random) sequence of measurement directions,

$$
\mathrm{E}\left(\kappa_{d-1} \lambda\left|\hat{\varphi}_{\gamma}^{\lambda}\left(L_{0}, \eta\right)\right|\right)^{3} \leq \omega_{d-1}^{3} \bar{\psi}_{\gamma}^{3} .
$$


Proof. Since E $N=\kappa_{d-1} \lambda$, we obtain, using Wald's identity,

$$
\mathrm{E} \kappa_{d-1} \lambda \hat{\varphi}_{\gamma}^{\lambda}\left(L_{0}, \eta\right)=\mathrm{E} \sum_{j=1}^{N} \hat{\varphi}_{\gamma}^{\lambda}\left(L_{0}, \eta\right)=\mathrm{E} \hat{\varphi}_{\gamma}^{\lambda}(\eta)=\sum_{i=1}^{n} C \varphi\left(\xi_{i}\right) \psi_{\gamma}\left(\eta, \xi_{i}\right) \Delta_{i},
$$

which yields the first claim. With

$$
\mathrm{E} Y_{i}\left(L_{0}\right) Y_{j}\left(L_{0}\right)=\mathrm{P}\left(L_{0} \cap \xi_{i}^{\perp} \cap W \neq \varnothing \text { and } L_{0} \cap \xi_{j}^{\perp} \cap W \neq \varnothing\right)=Q\left(H_{i, j}\right),
$$

we obtain

$$
\begin{aligned}
\mathrm{E}\left(\hat{\varphi}_{\gamma}^{\lambda}\left(L_{0}, \eta\right)\right)^{2} & =\mathrm{E}\left(\sum_{i=1}^{n} \tilde{Y}_{i}^{\lambda}\left(L_{0}\right) \psi_{\gamma}\left(\eta, \xi_{i}\right) \Delta_{i}\right)^{2} \\
& =\frac{1}{\kappa_{d-1}^{2} \lambda^{2}} \sum_{i=1}^{n} \sum_{j=1}^{n} \psi_{\gamma}\left(\eta, \xi_{i}\right) \psi_{\gamma}\left(\eta, \xi_{j}\right) \Delta_{i} \Delta_{j} Q\left(H_{i, j}\right)
\end{aligned}
$$

For the third moment,

$$
\mathrm{E}\left|\kappa_{d-1} \lambda \hat{\varphi}_{\gamma}^{\lambda}\left(L_{0}, \eta\right)\right|^{3}=\mathrm{E}\left|\sum_{i=1}^{n} Y_{i}\left(L_{0}\right) \psi_{\gamma}\left(\eta, \xi_{i}\right) \Delta_{i}\right|^{3} \leq \bar{\psi}_{\gamma}^{3} \sum_{i=1}^{n} \sum_{j=1}^{n} \sum_{k=1}^{n} \Delta_{i} \Delta_{j} \Delta_{k}=\omega_{d-1}^{3} \bar{\psi}_{\gamma}^{3} .
$$

Remark. Equation (1) is equivalent to $Q\left(H_{1}\right)=C \varphi\left(\xi_{1}\right)$, so we can replace $Q\left(H_{1}\right)$ accordingly in the theorem above.

Corollary 1. Let the conditions of Theorem 5 hold. If the measurement directions $\xi_{1}, \ldots, \xi_{n}$ are i.i.d. with common density $\zeta$ and independent of $\Xi$, then

$$
\begin{aligned}
& \mathrm{E}\left(\kappa_{d-1} \lambda \hat{\varphi}_{\gamma}^{\lambda}\left(L_{0}, \eta\right)\right)= 2 n \int_{\left(S^{d-1}\right)^{n}} \psi_{\gamma}\left(\eta, v_{1}\right)\left|c\left(v_{1} \mid v_{1}, \ldots, v_{n}\right)\right|_{d-1} Q\left(H_{v_{1}}\right) \\
& \times \zeta\left(v_{1}\right) \cdots \zeta\left(v_{n}\right) \mathrm{d}\left(v_{1}, \ldots, v_{n}\right), \\
& \mathrm{E}\left(\kappa_{d-1} \lambda \hat{\varphi}_{\gamma}^{\lambda}\left(L_{0}, \eta\right)\right)^{2}= n \mathrm{E}\left[\psi_{\gamma}^{2}\left(\eta, \xi_{1}\right) \Delta_{1}^{2} Y_{1}\left(L_{0}\right)\right] \\
&+n(n-1) \mathrm{E}\left[\psi_{\gamma}\left(\eta, \xi_{1}\right) \psi_{\gamma}\left(\eta, \xi_{2}\right) \Delta_{1} \Delta_{2} Y_{1}\left(L_{0}\right) Y_{2}\left(L_{0}\right)\right],
\end{aligned}
$$

with

$$
\begin{gathered}
\mathrm{E}\left[\psi_{\gamma}^{2}\left(\eta, \xi_{1}\right) \Delta_{1}^{2} Y_{1}\left(L_{0}\right)\right]=4 \int_{\left(S^{d-1}\right)^{n}} \psi_{\gamma}\left(\eta, v_{1}\right)^{2}\left|c\left(v_{1} \mid v_{1}, \ldots, v_{n}\right)\right|_{d-1}^{2} Q\left(H_{v_{1}}\right) \\
\times \zeta\left(v_{1}\right) \cdots \zeta\left(v_{n}\right) \mathrm{d}\left(v_{1}, \ldots, v_{n}\right),
\end{gathered}
$$

$\mathrm{E}\left[\psi_{\gamma}\left(\eta, \xi_{1}\right) \psi_{\gamma}\left(\eta, \xi_{2}\right) \Delta_{1} \Delta_{2} Y_{1}\left(L_{0}\right) Y_{2}\left(L_{0}\right)\right]$

$$
\begin{gathered}
=4 \int_{\left(S^{d-1}\right)^{n}} \psi_{\gamma}\left(\eta, v_{1}\right) \psi_{\gamma}\left(\eta, v_{2}\right)\left|c\left(v_{1} \mid v_{1}, \ldots, v_{n}\right)\right|_{d-1}\left|c\left(v_{2} \mid v_{1}, \ldots, v_{n}\right)\right|_{d-1} \\
\times Q\left(H_{v_{1}, v_{2}}\right) \zeta\left(v_{1}\right) \cdots \zeta\left(v_{n}\right) \mathrm{d}\left(v_{1}, \ldots, v_{n}\right) .
\end{gathered}
$$

Here, $|\cdot|_{d-1}$ denotes the $(d-1)$-dimensional Hausdorff measure, and $c\left(v_{1} \mid v_{1}, \ldots, v_{n}\right)$ is the Voronoi cell at $v_{1}$ induced by the points $v_{1}, \ldots, v_{n},-v_{1}, \ldots,-v_{n}$. 
Proof. We calculate

$$
\begin{aligned}
\mathrm{E}\left(\kappa_{d-1} \lambda \hat{\varphi}_{\gamma}^{\lambda}\left(L_{0}, \eta\right)\right) & =\kappa_{d-1} \lambda \mathrm{E} \sum_{i=1}^{n} \tilde{Y}_{i}^{\lambda}\left(L_{0}\right) \psi_{\gamma}\left(\eta, \xi_{i}\right) \Delta_{i} \\
& =n \mathrm{E} Y_{1}\left(L_{0}\right) \psi_{\gamma}\left(\eta, \xi_{1}\right) \Delta_{1} \\
& =n \int_{\left(S^{d-1}\right)^{n}} \mathrm{E}\left[\psi_{\gamma}\left(\eta, \xi_{1}\right) \Delta_{1} Y_{1}\left(L_{0}\right) \mid \xi_{1}=v_{1}, \ldots, \xi_{n}=v_{n}\right] \\
& \times \zeta\left(v_{1}\right) \cdots \zeta\left(v_{n}\right) \mathrm{d}\left(v_{1}, \ldots, v_{n}\right) \\
& \int_{\left(S^{d-1}\right)^{n}} \psi_{\gamma}\left(\eta, v_{1}\right)\left|c\left(v_{1} \mid v_{1}, \ldots, v_{n}\right)\right|_{d-1} Q\left(H_{v_{1}}\right) \\
& \times \zeta\left(v_{1}\right) \cdots \zeta\left(v_{n}\right) \mathrm{d}\left(v_{1}, \ldots, v_{n}\right)
\end{aligned}
$$

and, for the second moment,

$$
\begin{aligned}
\mathrm{E}\left(\kappa_{d-1} \lambda \hat{\varphi}_{\gamma}^{\lambda}\left(L_{0}, \eta\right)\right)^{2}= & n \sum_{j=1}^{n} \mathrm{E}\left[\psi_{\gamma}\left(\eta, \xi_{1}\right) \psi_{\gamma}\left(\eta, \xi_{j}\right) \Delta_{1} \Delta_{j} Y_{1}\left(L_{0}\right) Y_{j}\left(L_{0}\right)\right] \\
= & n \mathrm{E}\left[\psi_{\gamma}^{2}\left(\eta, \xi_{1}\right) \Delta_{1}^{2} Y_{1}\left(L_{0}\right)\right] \\
& +n(n-1) \mathrm{E}\left[\psi_{\gamma}\left(\eta, \xi_{1}\right) \psi_{\gamma}\left(\eta, \xi_{2}\right) \Delta_{1} \Delta_{2} Y_{1}\left(L_{0}\right) Y_{2}\left(L_{0}\right)\right] .
\end{aligned}
$$

With

$$
\begin{aligned}
& \mathrm{E}\left[\psi_{\gamma}\left(\eta, \xi_{1}\right) \psi_{\gamma}\left(\eta, \xi_{2}\right) \Delta_{1} \Delta_{2} Y_{1}\left(L_{0}\right) Y_{2}\left(L_{0}\right)\right] \\
& =\int_{\left(S^{d-1}\right)^{n}} \mathrm{E}\left[\psi_{\gamma}\left(\eta, \xi_{1}\right) \psi_{\gamma}\left(\eta, \xi_{2}\right) \Delta_{1} \Delta_{2} Y_{1}\left(L_{0}\right) Y_{2}\left(L_{0}\right) \mid \xi_{1}=v_{1}, \ldots, \xi_{n}=v_{n}\right] \\
& \times \zeta\left(v_{1}\right) \cdots \zeta\left(v_{n}\right) \mathrm{d}\left(v_{1}, \ldots, v_{n}\right) \\
& =4 \int_{\left(S^{d-1}\right)^{n}} \psi_{\gamma}\left(\eta, v_{1}\right) \psi_{\gamma}\left(\eta, v_{2}\right)\left|c\left(v_{1} \mid v_{1}, \ldots, v_{n}\right)\right|_{d-1}\left|c\left(v_{2} \mid v_{1}, \ldots, v_{n}\right)\right|_{d-1} \\
& \times Q\left(H_{v_{1}, v_{2}}\right) \zeta\left(v_{1}\right) \cdots \zeta\left(v_{n}\right) \mathrm{d}\left(v_{1}, \ldots, v_{n}\right),
\end{aligned}
$$

the second expression is shown. The calculation for $\mathrm{E}\left[\psi_{\gamma}^{2}\left(\eta, \xi_{1}\right) \Delta_{1}^{2} Y_{1}\left(L_{0}\right)\right]$ can be done analogously.

In the next corollary, we consider the following systematic sampling approach. Suppose that we have a (deterministic) sequence of points $\widetilde{\xi}_{1}, \ldots, \tilde{\xi}_{n}$ on the sphere. Furthermore, let the rotation $\Theta$ be uniformly distributed in the group $\mathrm{SO}_{d}$ of orientation-preserving rotations on $\mathbb{R}^{d}$ and independent of $\Xi$. Then we consider the measurement points $\left(\xi_{1}, \ldots, \xi_{n}\right)=$ $\left(\Theta \tilde{\xi}_{1}, \ldots, \Theta \tilde{\xi}_{n}\right)$.

Corollary 2. Let the conditions of Theorem 5 hold. Suppose that measurement directions $\xi_{1}, \ldots, \xi_{n}$ are chosen by systematic sampling as described above. Then

$$
\begin{aligned}
\mathrm{E}\left(\kappa_{d-1} \lambda \hat{\varphi}_{\gamma}^{\lambda}\left(L_{0}, \eta\right)\right) & =\varphi_{\gamma}(\eta), \\
\mathrm{E}\left(\kappa_{d-1} \lambda \hat{\varphi}_{\gamma}^{\lambda}\left(L_{0}, \eta\right)\right)^{2} & =\sum_{i=1}^{n} \sum_{j=1}^{n} \int_{\mathrm{SO}_{d}} \psi_{\gamma}\left(\eta, \theta \tilde{\xi}_{i}\right) \psi_{\gamma}\left(\eta, \theta \tilde{\xi}_{j}\right) Q\left(H_{\theta \tilde{\xi}_{i}, \theta \tilde{\xi}_{j}}\right) \mathrm{d} \theta \Delta_{i} \Delta_{j},
\end{aligned}
$$

where $\mathrm{d} \theta$ denotes the Haar probability measure on $\mathrm{SO}_{d}$. 
Proof. With the rotation $\Theta$ introduced above and Theorem 6, we calculate

$$
\begin{aligned}
\mathrm{E}\left(\kappa_{d-1} \lambda \hat{\varphi}_{\gamma}^{\lambda}\left(L_{0}, \eta\right)\right) & =\int_{\mathrm{SO}_{d}} \mathrm{E}\left[\left(\kappa_{d-1} \lambda \hat{\varphi}_{\gamma}^{\lambda}\left(L_{0}, \eta\right)\right) \mid \Theta=\theta\right] \mathrm{d} \theta \\
& =\int_{\mathrm{SO}_{d}} \sum_{i=1}^{n} C \varphi\left(\theta \tilde{\xi}_{i}\right) \psi_{\gamma}\left(\eta, \theta \tilde{\xi}_{i}\right) \Delta_{i} \mathrm{~d} \theta \\
& =\sum_{i=1}^{n} \int_{\mathrm{SO}_{d}} C \varphi\left(\theta \tilde{\xi}_{i}\right) \psi_{\gamma}\left(\eta, \theta \tilde{\xi}_{i}\right) \mathrm{d} \theta \Delta_{i} \\
& =\sum_{i=1}^{n} \frac{1}{\omega_{d-1}} \varphi_{\gamma}(\eta) \Delta_{i} \\
& =\varphi_{\gamma}(\eta)
\end{aligned}
$$

using (2). Repeating these arguments and using the corresponding formula from Theorem 6 lead to the claim for the second moment.

In the following we derive specific formulae for the term $Q\left(H_{1,2}\right)$, which appears in Theorem 6 , for the most interesting dimensions $d=2,3$. It is convenient to define the set $H_{1,2}(v)=H_{1,2}(-v)$ as the subset of $H_{1,2}$ containing the lines with direction $v \in S^{d-1}$. This can be regarded as a subset of $\nu^{\perp}$, and, thus, we obtain

$$
\begin{aligned}
Q\left(H_{1,2}\right) & =\int_{S^{d-1}} \mathrm{P}\left(L_{0} \cap \xi_{1} \cap W \neq \varnothing, L_{0} \cap \xi_{2} \cap W \neq \varnothing \mid L_{0} \in \Lambda_{d}(v)\right) \varphi(v) \mathrm{d} v \\
& =\int_{S^{d-1}} \frac{\left|H_{1,2}(v)\right|_{d-1}}{\kappa_{d-1}} \varphi(v) \mathrm{d} \nu,
\end{aligned}
$$

where $\Lambda_{d}(v)$ is the set of all lines in $\mathbb{R}^{d}$ with direction $v$.

\subsection{Two-dimensional case}

For the two-dimensional case, we introduce some special notation which seems to be more common. Instead of even functions on the sphere, we consider densities on the interval $[0, \pi]$, where each value corresponds to an angle between a vector and the $x$-axis. For simplicity, all functions, in particular the density $\varphi$, are defined to be $\pi$-periodic. For unit vectors, we use the notation $\vec{v}:=(\cos v, \sin v)^{\top}$, where $v \in[0,2 \pi]$, and, for the line through $\vec{v}$ (and o), we write $\bar{v}$.

Furthermore, in this section, $\xi_{i j}$ denotes the angle between the $x$-axis and the test lines instead of the vectors orthogonal to the test hyperplanes, i.e. instead of counting the intersections of $\Xi$ and $\vec{v}^{\perp}$, we define $Y_{\text {in }}:=\#\left(\Xi \cap \bar{\xi}_{\text {in }} \cap W\right)$. Thus, the rose of intersections is the sine transform of $\varphi$ :

$$
g(x)=\lambda S \varphi(x)=\lambda \int_{0}^{\pi}|\sin (x-t)| \varphi(t) \mathrm{d} t .
$$

Because of (7), we need to calculate $\left|H_{1,2}(v)\right|_{1}$. As can be seen in Figure 2, for one line, the measure of this set is $\left|H_{1}(v)\right|_{1}=2 \sin \left|v-\xi_{1}\right|$. Similarly, if we have two lines $\bar{\xi}_{1}$ and $\bar{\xi}_{2}$, we obtain

$$
\left|H_{1,2}(v)\right|_{1}=\left|H_{1}(v) \cap H_{2}(v)\right|_{1}=2 \sin \left(\min \left\{\left|v-\xi_{1}\right|,\left|v-\xi_{2}\right|\right\}\right) .
$$




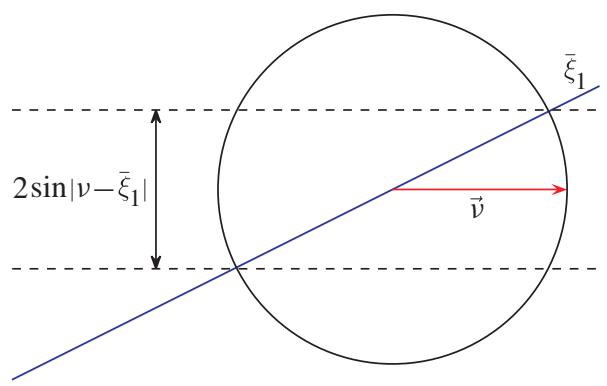

FIGURE 2: Example of lines with direction $\vec{v}$ hitting one line $\bar{\xi}_{1}$.

Assuming that $\xi_{1} \leq \xi_{2}$, this leads to the formula

$$
\begin{aligned}
Q\left(H_{1,2}\right)= & \frac{1}{2} \int_{0}^{\pi}\left|H_{1,2}(v)\right|_{1} \varphi(v) \mathrm{d} v \\
= & \int_{0}^{\pi} \sin \left(\min \left\{\left|v-\xi_{1}\right|,\left|v-\xi_{2}\right|\right\}\right) \varphi(v) \mathrm{d} v \\
= & \int_{0}^{\left(\xi_{2}-\xi_{1}\right) / 2} \sin v\left(\varphi\left(\xi_{1}+v\right)+\varphi\left(\xi_{2}-v\right)\right) \mathrm{d} v \\
& +\int_{0}^{\pi / 2-\left(\xi_{2}-\xi_{1}\right) / 2} \sin v\left(\varphi\left(\xi_{2}+v\right)+\varphi\left(\xi_{1}-v\right)\right) \mathrm{d} v .
\end{aligned}
$$

Example. For uniformly distributed directions $(\varphi(x)=1 / \pi)$, the formulae for $Q\left(H_{1, \ldots, m}\right)$ can be simplified to

$$
Q\left(H_{1}\right)=S \varphi\left(\xi_{1}\right)=\frac{2}{\pi} \int_{0}^{\pi / 2} \sin v \mathrm{~d} v=\frac{2}{\pi}
$$

and

$$
\begin{aligned}
Q\left(H_{1,2}\right) & =\frac{2}{\pi}\left(\int_{0}^{\left(\xi_{2}-\xi_{1}\right) / 2} \sin v \mathrm{~d} v+\int_{0}^{\pi / 2-\left(\xi_{2}-\xi_{1}\right) / 2} \sin v \mathrm{~d} v\right) \\
& =\frac{2}{\pi}\left[2-\sqrt{2} \cos \left(\frac{\pi}{4}-\frac{\xi_{2}-\xi_{1}}{2}\right)\right],
\end{aligned}
$$

where we used an addition rule for the cosine.

\subsection{Three-dimensional case}

Here, $H_{1,2}(v)$ is $\xi_{1}^{\perp} \cap b_{1}(\mathbf{o})$ projected onto $v^{\perp}$ intersected with $\xi_{2}^{\perp} \cap b_{1}(\mathbf{o})$ projected onto $v^{\perp}$, i.e.

$$
H_{1,2}(v)=\pi_{v^{\perp}}\left(\xi_{1}^{\perp} \cap b_{1}(\mathbf{o})\right) \cap \pi_{v^{\perp}}\left(\xi_{2}^{\perp} \cap b_{1}(\mathbf{o})\right),
$$

where $\pi_{v \perp}$ denotes the projection onto the plane $v^{\perp}$; see Figure 3 for an example. This means that $H_{1,2}(v)$ is the intersection of two ellipses with major axis length 1 and minor axis lengths $\left|\left\langle\nu, \xi_{1}\right\rangle\right|$ and $\left|\left\langle\nu, \xi_{2}\right\rangle\right|$, respectively. The angle between the major axes is the same as the angle between $\pi_{v^{\perp}} \xi_{1}$ and $\pi_{v^{\perp}} \xi_{2}$ and can be determined easily. For the calculation of the intersection area, see Appendix A. Then

$$
Q\left(H_{1,2}\right)=\int_{S^{2}} \frac{\left|H_{1,2}(\nu)\right|_{2}}{\pi} \varphi(\nu) \mathrm{d} \nu .
$$




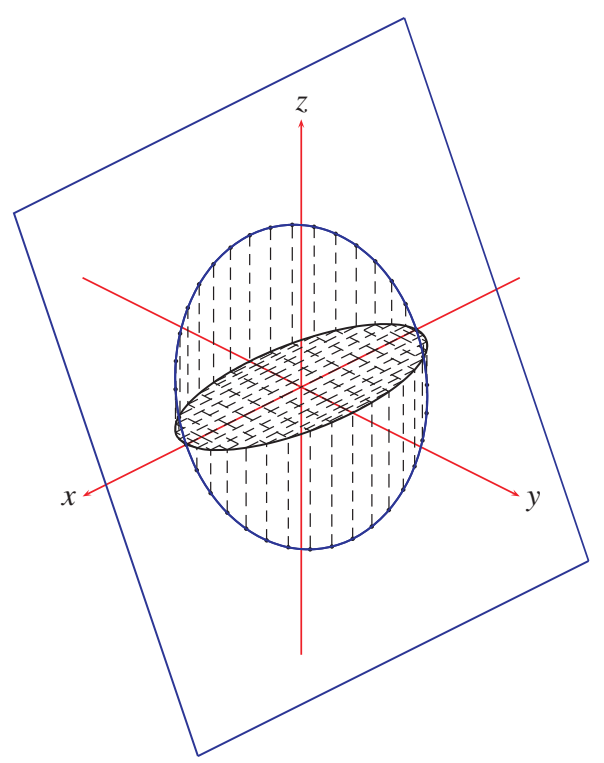

FIGURE 3: Sketch of the projection of $\xi_{1}^{\perp} \cap b_{1}$ (o) onto $v^{\perp}$. Here we have $v=(0,0,1)^{\top}$ and $\xi_{1}=(0,1 / \sqrt{5}, 2 / \sqrt{5})^{\top}$.

\subsection{Application: testing the directional distribution}

In this section we present some tests of the directional distribution of a Poisson line process $\Xi$, i.e. we want to test the hypothesis $H_{0}: \varphi=\varphi_{0}$ versus $H_{1}: \varphi \neq \varphi_{0}$ for some density $\varphi_{0}$ on the unit sphere, where the uniform distribution (i.e. isotropy) is of course the most interesting. We denote by $\mathrm{P}_{0}$ the probability measure under the null hypothesis, and analogously we write $\mathrm{E}_{0}, \operatorname{cov}_{0}$, and $Q_{0}$.

For simplicity, we restrict to the case when the measurement directions $\xi_{1}, \ldots, \xi_{n}$ are deterministic.

As shown in Theorem 5, an asymptotic test on the density in one point $\eta$ can be constructed with the asymptotically standard Gaussian distributed random variable

$$
\frac{\hat{\varphi}_{\gamma}^{\lambda}(\eta)-\kappa_{d-1} \lambda \mathrm{E}_{0} \hat{\varphi}_{\gamma}^{\lambda}\left(L_{0}, \eta\right)}{\sqrt{\kappa_{d-1} \lambda \mathrm{E}_{0} \hat{\varphi}_{\gamma}^{\lambda}\left(L_{0}, \eta\right)^{2}}} \stackrel{\mathrm{D}}{\rightarrow} \mathrm{N}(0,1) \quad \text { as } \varrho \rightarrow \infty .
$$

This test is of course only applicable for large $\varrho$. One problem of this test is the choice of $\eta$. If several independent realizations of the Poisson line process are available, an asymptotic $\chi^{2}$-test can be constructed using (10) with a different $\eta$ for each realization.

If only one realization can be observed, several directions (denoted by $\eta_{1}, \ldots, \eta_{m}$ ) can be taken into account with the following approach. The vector of the random variables

$$
X_{i}:=\frac{\hat{\varphi}_{\gamma}^{\lambda}\left(\eta_{i}\right)-\kappa_{d-1} \lambda \mathrm{E}_{0} \hat{\varphi}_{\gamma}^{\lambda}\left(L_{0}, \eta_{i}\right)}{\sqrt{\kappa_{d-1} \lambda \mathrm{E}_{0} \hat{\varphi}_{\gamma}^{\lambda}\left(L_{0}, \eta_{i}\right)^{2}}}, \quad i=1, \ldots, m,
$$

has an asymptotic multivariate normal distribution, which can easily be seen with Lemma 6, since, for any linear combination, a central limit theorem holds. Furthermore, their expectation 
is 0 and the covariance is independent of $\lambda$, since

$$
\begin{aligned}
\operatorname{cov}_{0}\left(X_{i}, X_{j}\right) & =\frac{\operatorname{cov}_{0}\left(\hat{\varphi}_{\gamma}^{\lambda}\left(\eta_{i}\right), \hat{\varphi}_{\gamma}^{\lambda}\left(\eta_{j}\right)\right)}{\kappa_{d-1} \lambda \sqrt{\mathrm{E}_{0} \hat{\varphi}_{\gamma}^{\lambda}\left(L_{0}, \eta_{i}\right)^{2}} \sqrt{\mathrm{E}_{0} \hat{\varphi}_{\gamma}^{\lambda}\left(L_{0}, \eta_{j}\right)^{2}}} \\
& =\frac{\sum_{k=1}^{n} \sum_{l=1}^{n} \psi_{\gamma}\left(\eta_{i}, \xi_{k}\right) \psi_{\gamma}\left(\eta_{j}, \xi_{l}\right) \Delta_{k} \Delta_{l} Q_{0}\left(H_{k, l}\right)}{\sqrt{\mathrm{E}_{0}\left(\kappa_{d-1} \lambda \hat{\varphi}_{\gamma}^{\lambda}\left(L_{0}, \eta_{i}\right)\right)^{2}} \sqrt{\mathrm{E}_{0}\left(\kappa_{d-1} \lambda \hat{\varphi}_{\gamma}^{\lambda}\left(L_{0}, \eta_{j}\right)\right)^{2}}},
\end{aligned}
$$

where we have used the fact that

$$
\begin{aligned}
\operatorname{cov}\left(\hat{\varphi}_{\gamma}^{\lambda}\left(\eta_{i}\right), \hat{\varphi}_{\gamma}^{\lambda}\left(\eta_{j}\right)\right) & =\operatorname{cov}\left(\sum_{k=1}^{n} \frac{Y_{k}}{\kappa_{d-1} \lambda} \psi_{\gamma}\left(\eta_{i}, \xi_{k}\right) \Delta_{k}, \sum_{l=1}^{n} \frac{Y_{l}}{\kappa_{d-1} \lambda} \psi_{\gamma}\left(\eta_{j}, \xi_{l}\right) \Delta_{l}\right) \\
& =\frac{1}{\kappa_{d-1}^{2} \lambda^{2}} \sum_{k=1}^{n} \sum_{l=1}^{n} \psi_{\gamma}\left(\eta_{i}, \xi_{k}\right) \psi_{\gamma}\left(\eta_{j}, \xi_{l}\right) \Delta_{k} \Delta_{l} \operatorname{cov}\left(Y_{k}, Y_{l}\right) \\
& =\frac{1}{\kappa_{d-1} \lambda} \sum_{k=1}^{n} \sum_{l=1}^{n} \psi_{\gamma}\left(\eta_{i}, \xi_{k}\right) \psi_{\gamma}\left(\eta_{j}, \xi_{l}\right) \Delta_{k} \Delta_{l} Q\left(H_{k, l}\right) .
\end{aligned}
$$

Thus, it follows from the Cramér-Wold theorem that the random variable $X^{\top} K_{X}^{-1} X$ is asymptotically $\chi_{m}^{2}$-distributed, where $X=\left(X_{1}, \ldots, X_{m}\right)^{\top}$ and $K_{X}$ is the covariance matrix of $X$ for some $\lambda>0$. The resulting rule for a level $\alpha$ test is to reject the null hypothesis if $X^{\top} K_{X}^{-1} X>\chi_{m, 1-\alpha}^{2}$.

This is most interesting when the null hypothesis is that the process is isotropic. Then, for $Q\left(H_{k, l}\right)$, we can use (8) in the two-dimensional case and (9) in the three-dimensional case with $\varphi \equiv 1 / 4 \pi$.

\section{Large deviations}

Another interesting asymptotic property of the estimator $\hat{\varphi}_{\gamma}^{\lambda}$ is the large deviation behavior. The following result is based on Cramér's theorem; see [2, Theorem I.4].

Theorem 7. Let $\varrho=1$. It holds that, for $a>\operatorname{E} \hat{\varphi}_{\gamma}^{\lambda}(\eta)$,

$$
\lim _{\lambda \rightarrow \infty} \frac{1}{\lambda} \log \mathrm{P}\left(\hat{\varphi}_{\gamma}^{\lambda}(\eta) \geq a\right)=-I(a),
$$

and, similarly, for $a<\mathrm{E} \hat{\varphi}_{\gamma}^{\lambda}(\eta)$,

$$
\lim _{\lambda \rightarrow \infty} \frac{1}{\lambda} \log \mathrm{P}\left(\hat{\varphi}_{\gamma}^{\lambda}(\eta) \leq a\right)=-I(a),
$$

with the rate function

$$
I(z)=\sup _{t \in \mathbb{R}}\left[z t-\kappa_{d-1}\left(m_{\widetilde{\varphi}_{\gamma}\left(L_{0}\right)}(t)-1\right)\right]
$$

for

$$
\tilde{\varphi}_{\gamma}\left(L_{0}\right)=\frac{1}{\kappa_{d-1}} \sum_{k=1}^{n} Y_{k}\left(L_{0}\right) \psi_{\gamma}\left(\eta, \xi_{k}\right) \Delta_{k},
$$

where $L_{0} \sim Q$ is a typical line and

$$
m_{\widetilde{\varphi}_{\gamma}\left(L_{0}\right)}(t)=\sum_{j=1}^{n} \sum_{1 \leq i_{1}<\cdots<i_{j} \leq n} \exp \left\{\frac{t}{\kappa_{d-1}} \sum_{k=1}^{j} \psi_{\gamma}\left(\eta, \xi_{i_{k}}\right) \Delta_{i_{k}}\right\} \mathrm{P}\left(Y_{i_{1}, \ldots, i_{j}}^{*}\left(L_{0}\right)=1\right) .
$$


Here $m_{X}(t)=\mathrm{E} \mathrm{e}^{t X}$ denotes the moment generating function of a random variable $X$ and

$$
Y_{i_{1}, \ldots, i_{j}}^{*}(\cdot)=\prod_{k \in\left\{i_{1}, \ldots, i_{j}\right\}} Y_{k}(\cdot) \prod_{k \in\left\{i_{1}, \ldots, i_{j}\right\}^{c}}\left(1-Y_{k}(\cdot)\right)
$$

is defined such that $Y_{i_{1}, \ldots, i_{j}}^{*}(l)=1$ if and only if $l \in \Lambda_{d}$ hits the test hyperplanes $\xi_{i_{1}}, \ldots, \xi_{i_{j}}$ (and no others) within W.

Proof. We use the idea with the compound Poisson distribution from Section 3 and additionally note that, for an integer $\lambda$ and i.i.d. random variables $N_{1}, \ldots, N_{\lambda}$ with $N_{1} \sim \operatorname{Poi}\left(\kappa_{d-1}\right)$, we have $\sum_{i=1}^{\lambda} N_{i} \stackrel{\mathrm{D}}{=} N$, as $N \sim \operatorname{Poi}\left(\kappa_{d-1} \lambda\right)$. Assuming that $N_{1}, \ldots, N_{\lambda}$ are independent of the i.i.d. random lines $L_{i j} \sim Q$ for $i, j \geq 1$, we obtain

$$
\hat{\varphi}_{\gamma}^{\lambda}(\eta) \stackrel{\mathrm{D}}{=} \sum_{i=1}^{\lambda} \sum_{j=1}^{N_{i}} \hat{\varphi}_{\gamma}^{\lambda}\left(L_{i j}, \eta\right)=\frac{1}{\lambda} \sum_{i=1}^{\lambda} \sum_{j=1}^{N_{i}} \frac{1}{\kappa_{d-1}} \sum_{k=1}^{n} Y_{k}\left(L_{i j}\right) \psi_{\gamma}\left(\eta, \xi_{k}\right) \Delta_{k} .
$$

Thus, $\hat{\varphi}_{\gamma}^{\lambda}(\eta)$ can be written as the average of $\lambda$ i.i.d. random variables.

To apply Cramér's theorem, it remains to calculate the moment generating function of $\sum_{j=1}^{N_{1}} X_{j}$, where

$$
X_{j}=\frac{1}{\kappa_{d-1}} \sum_{k=1}^{n} Y_{k}\left(L_{1 j}\right) \psi_{\gamma}\left(\eta, \xi_{k}\right) \Delta_{k}=\tilde{\varphi}_{\gamma}\left(L_{1 j}\right),
$$

which can be done with [11, Lemma 3.1]:

$$
\begin{aligned}
m_{\sum_{j=1}^{N_{1}} X_{j}}(t) & =m_{N_{1}}\left[\log m_{X_{1}}(t)\right] \\
& =\exp \left\{\kappa_{d-1}\left(\exp \left\{\log m_{X_{1}}(t)\right\}-1\right)\right\} \\
& =\exp \left\{\kappa_{d-1}\left(m_{X_{1}}(t)-1\right)\right\} .
\end{aligned}
$$

Thus, we arrive at (12) by calculating

$$
\begin{aligned}
m_{X_{1}}(t) & =\mathrm{Ee}^{t X_{1}} \\
& =\operatorname{E} \exp \left\{\frac{t}{\kappa_{d-1}} \sum_{i=1}^{n} Y_{i}\left(L_{0}\right) \psi_{\gamma}\left(\eta, \xi_{i}\right) \Delta_{i}\right\} \\
& =\mathrm{E} \sum_{j=1}^{n} \sum_{1 \leq i_{1}<\cdots<i_{j} \leq n} \exp \left\{\frac{t}{\kappa_{d-1}} \sum_{k=1}^{j} \psi_{\gamma}\left(\eta, \xi_{i_{k}}\right) \Delta_{i_{k}}\right\} \mathbf{1}\left\{Y_{i_{1}, \ldots, i_{j}}^{*}\left(L_{0}\right)=1\right\} \\
& =\sum_{j=1}^{n} \sum_{1 \leq i_{1}<\cdots<i_{j} \leq n} \exp \left\{\frac{t}{\kappa_{d-1}} \sum_{k=1}^{j} \psi_{\gamma}\left(\eta, \xi_{i_{k}}\right) \Delta_{i_{k}}\right\} \mathrm{P}\left(Y_{i_{1}, \ldots, i_{j}}^{*}\left(L_{0}\right)=1\right) .
\end{aligned}
$$

Note that some summands in (12) are 0 because it is not possible that one line hits an arbitrary set of test hyperplanes within the observation window, only sequences of neighboring hyperplanes can be intersected.

\section{Numerical simulation results}

For applications, it is important to know how good the normal approximation of the test statistics in Section 3.3 is at a certain radius and intensity. Here, we analyze simulation data 
TABLE 1: Rejection rates for level 0.05 Kolmogorov-Smirnoff (KS) and Shapiro-Wilk (SK) tests at different radii and measurement point sets. The hypothesis of isotropy is being tested for an isotropic sample. For all reconstructions, $\gamma=0.2$.

\begin{tabular}{rcccccccc}
\hline \multirow{2}{*}{ Radius } & \multicolumn{2}{c}{49 points } & & \multicolumn{2}{c}{100 points } & & \multicolumn{2}{c}{225 points } \\
\cline { 2 - 3 } \cline { 7 - 8 } & KS test & SW test & & KS test & SW test & & KS test & SW test \\
\hline 5 & 0.19 & 0.05 & & 0.46 & 0.15 & & 0.28 & 0.02 \\
10 & 0.13 & 0.1 & & 0.13 & 0.07 & & 0.11 & 0.08 \\
15 & 0.08 & 0.07 & & 0.09 & 0.04 & & 0.08 & 0.05 \\
20 & 0.07 & 0.07 & & 0.06 & 0.08 & & 0.08 & 0.06 \\
\hline
\end{tabular}

with different observation window radii for the most important case, the uniform distribution (i.e. isotropy). For this, we have simulated 100000 independent copies of a stationary isotropic Poisson line process with intensity 1 . For different observation window radii, we have analyzed the deviation of the distribution of the $X_{i}$ as defined in (11) from the standard Gaussian distribution, where the expectation values in the definition have been computed numerically using Theorem 6 and the results from Section 3.2. To evaluate the results, we have split our simulation data into 100 groups of size 1000. For each group, we have conducted a level 0.05 Kolmogorov-Smirnoff and Shapiro-Wilk test. In Table 1, the rejection rates are listed for different radii at the exemplary point $(-0.40382,-0.23972,0.88287)^{\top}$, which we have selected from 20 random points because the values seem representative. We consider three of the point sets in [16] as measurement points. At a radius between 15 and 20, the approximation seems to be reasonably good with $\gamma=0.2$. For differing values of $\gamma$, the convergence gets slower.

\section{Appendix A. Intersection area of two ellipses}

Here we calculate the area of the intersection of two ellipses within the unit disk in $\mathbb{R}^{2}$ needed in Sections 3.2 and 3.3. Our results allow the numerical calculation of $Q\left(H_{1,2}\right)$. It suffices to consider centered ellipses with length 1 and arbitrary widths not exceeding 1 . We denote the angle between their major axes by $\theta$. For the first ellipse $E_{1}$, we assume without loss of generality that its major axis is the first coordinate axis and it has a width $a_{1}$ with $0<a_{1} \leq 1$, i.e.

$$
E_{1}=\left\{(x, y)^{\top}: x^{2}+\frac{y^{2}}{a_{1}^{2}} \leq 1\right\} .
$$

The other ellipse $E_{2}$ also has length 1 and the width is denoted by $a_{2}$, assuming again that $0<a_{2} \leq 1$. Its major axis has an angle $\theta$ with respect to the first coordinate axis. Formally,

$$
E_{2}=\left\{(x, y)^{\top}:(x \cos \theta+y \sin \theta)^{2}+\frac{(x \sin \theta-y \cos \theta)^{2}}{a_{2}^{2}} \leq 1\right\} ;
$$

see also Figure 4 .

For the calculation of $\left|E_{1} \cap E_{2}\right|_{2}$, we consider the ellipses stretched by the factor $1 / a_{1}$ in the $y$-direction. We denote this linear map by $Y_{1 / a_{1}}$. This operation depicts $E_{1}$ onto the unit circle, and $E_{2}$ onto the ellipse $\tilde{E}_{2}=Y_{1 / a_{1}} E_{2}$. Our calculation consists of two parts, namely the determination of the lengths of the axes of $\tilde{E}_{2}$ (Lemma 7 below), and the calculation of the intersection area of a centered ellipse and the unit disk (Lemma 8 below). 


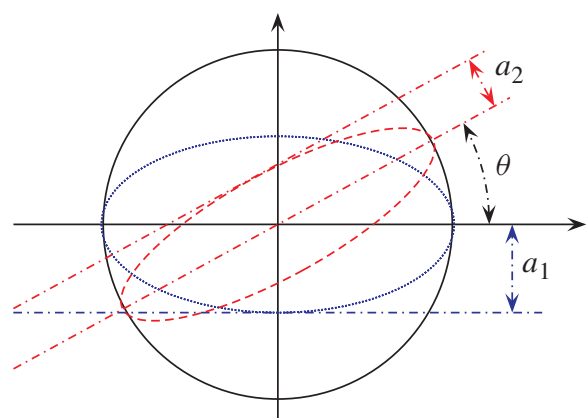

Figure 4: Draft of the two ellipses under consideration, $E_{1}$ (dotted ellipse) and $E_{2}$ (dashed ellipse).

Lemma 7. The square lengths of the axes of the ellipse $\tilde{E}_{2}=Y_{1 / a_{1}} E_{2}$, where $E_{2}$ is defined in (13), are

$$
\begin{aligned}
\lambda_{1,2}= & \frac{1}{2}\left[\left(1+\frac{a_{2}^{2}}{a_{1}^{2}}\right) \cos ^{2} \theta+\left(\frac{1}{a_{1}^{2}}+a_{2}^{2}\right) \sin ^{2} \theta\right] \\
& \pm \sqrt{\frac{1}{4}\left[\left(1+\frac{a_{2}^{2}}{a_{1}^{2}}\right) \cos ^{2} \theta+\left(\frac{1}{a_{1}^{2}}+a_{2}^{2}\right) \sin ^{2} \theta\right]^{2}-\frac{a_{2}^{2}}{a_{1}^{2}}}
\end{aligned}
$$

Proof. The ellipse $\tilde{E}_{2}$ can be written as the result of a linear map applied to the unit disk:

$$
\begin{aligned}
\tilde{E}_{2} & =Y_{1 / a_{1}} R_{\theta} Y_{a_{2}} b_{1}(\mathbf{o}) \\
& =\left(\begin{array}{cc}
1 & 0 \\
0 & 1 / a_{1}
\end{array}\right)\left(\begin{array}{cc}
\cos \theta & -\sin \theta \\
\sin \theta & \cos \theta
\end{array}\right)\left(\begin{array}{cc}
1 & 0 \\
0 & a_{2}
\end{array}\right) b_{1}(\mathbf{o}) \\
& =\left(\begin{array}{cc}
\cos \theta & -a_{2} \sin \theta \\
\frac{1}{a_{1}} \sin \theta & \frac{a_{2}}{a_{1}} \cos \theta
\end{array}\right) b_{1}(\mathbf{o}),
\end{aligned}
$$

where $R_{\theta}$ denotes the rotation about the angle $\theta$. With a singular value decomposition of the latter matrix we can find the desired lengths of the axes.

A few remarks are in order.

- It can be shown that the constants $\lambda_{1}$ and $\lambda_{2}$ in Lemma 7 are always positive.

- $\lambda_{1}=\lambda_{2}$ holds if and only if $a_{1}=a_{2}=1$ or we have $\theta=0$ and $a_{1}=a_{2}$. In both cases, the ellipse $\tilde{E}_{2}$ is a circle of radius 1 , which leads to $\lambda_{1}=\lambda_{2}=1$.

For the main result of this section, we need one more lemma.

Lemma 8. For a centered ellipse $E$ with axis lengths $a$ and $b$, where $1 \leq a$ and $0<b \leq 1$, it holds that

$$
\left|E \cap b_{1}(\mathbf{0})\right|_{2}=2 \arccos \sqrt{\frac{a^{2}\left(1-b^{2}\right)}{a^{2}-b^{2}}}+2 a b \arcsin \sqrt{\frac{1-b^{2}}{a^{2}-b^{2}}} .
$$

Proof. The claim follows by calculating the intersection points of the boundary of $E$ and the unit sphere and respective areas. 
Combining these two lemmas leads to the main result of this section.

Proposition 1. Let $E_{1}$ and $E_{2}$ be two centered ellipses with major axis lengths 1 and minor axis lengths $a_{1}$ and $a_{2}$, respectively $\left(0<a_{1}, a_{2} \leq 1\right)$, where the angle between the two major axes is denoted by $\theta$ (see Figure 4).

Then, for $a_{1}=a_{2}=1$ and for $a_{1}=a_{2}$ and $\theta=0$, we have $\left|E_{1} \cap E_{2}\right|_{2}=a_{1} \pi$. Otherwise,

$$
\left|E_{1} \cap E_{2}\right|_{2}=2 a_{1}\left(\arccos \sqrt{\frac{\lambda_{1}\left(1-\lambda_{2}\right)}{\lambda_{1}-\lambda_{2}}}+\sqrt{\lambda_{1} \lambda_{2}} \arcsin \sqrt{\frac{1-\lambda_{2}}{\lambda_{1}-\lambda_{2}}}\right),
$$

where

$$
\begin{aligned}
\lambda_{1,2}= & \frac{1}{2}\left[\left(1+\frac{a_{2}^{2}}{a_{1}^{2}}\right) \cos ^{2} \theta+\left(\frac{1}{a_{1}^{2}}+a_{2}^{2}\right) \sin ^{2} \theta\right] \\
& \pm \sqrt{\frac{1}{4}\left[\left(1+\frac{a_{2}^{2}}{a_{1}^{2}}\right) \cos ^{2} \theta+\left(\frac{1}{a_{1}^{2}}+a_{2}^{2}\right) \sin ^{2} \theta\right]^{2}-\frac{a_{2}^{2}}{a_{1}^{2}}}
\end{aligned}
$$

\section{Appendix B. Reconstruction kernels for the cosine (and spherical Radon) transform in arbitrary dimensions}

In this section we derive reconstruction kernels for the cosine transform in arbitrary dimensions $d \geq 3$. For $d=2,3$, closed expressions can be found in [10]. At first, we construct a reconstruction kernel for the so-called spherical Radon transform, which is defined for even functions $f$ as

$$
R f(\eta)=\frac{1}{\omega_{d-2}} \int_{S^{d-1} \cap \eta^{\perp}} f(\xi) \mathrm{d} \xi, \quad \eta \in S^{d-1},
$$

and use its relatedness to the cosine transform to arrive at the desired reconstruction kernel. Basically, we generalize the procedure from [10] to arbitrary dimensions $d \geq 3$, concentrating on the Gaussian mollifier.

We begin with some facts about the spherical Radon transform. It commutes with rotations (see [3, Lemma C.2.7]), i.e.

$$
R\left(T_{A} f(\eta)\right)=T_{A} R f(\eta), \quad A \in \mathrm{SO}_{d},
$$

with $T_{A} f(\xi):=f\left(A^{-1} \xi\right)$. Therefore, it is sufficient to construct a kernel for only one fixed point $\xi_{0} \in S^{d-1}$. We demand that the mollifier depends only on the geodesic distance $d(\xi, \eta)$ between $\xi, \eta \in S^{d-1}$, and not on $\xi$ and $\eta$ themselves. Thus, the choice of $\xi_{0}$ to be $e_{d}$, the $d$ th vector of the standard basis of $\mathbb{R}^{d}$, has the result that $e_{\gamma}\left(\xi_{0}, \eta\right)$ depends only on the polar angle of $\eta$, denoted by $\theta$. For this, we will use the notation $e_{\gamma}(\theta):=e_{\gamma}\left(\xi_{0}, \eta\right)$.

For the construction of the reconstruction kernel, we will use the following result.

Theorem 8. (Cf. [3, pp. 432-434].) Let $f, g \in C^{1}\left(S^{d-1}\right)$ be rotationally symmetric functions with $R f=g$. Then

$$
f(\arccos t)=\frac{1}{(d-3) !} t\left(\frac{1}{t} \frac{\mathrm{d}}{\mathrm{d} t}\right)^{d-2} \int_{0}^{t} g(\arcsin x) x^{d-2}\left(t^{2}-x^{2}\right)^{(d-4) / 2} \mathrm{~d} x .
$$


To calculate the normalizing constant, we recall that we require $\int_{S^{d-1}} e_{\gamma}(\xi) \mathrm{d} \xi=1$ for all $\gamma>0$, which can be rewritten for rotationally symmetric mollifiers $e_{\gamma}(\theta)$ as

$$
2 \omega_{d-1} \int_{0}^{\pi / 2} e_{\gamma}(\theta)(\sin \theta)^{d-2} \mathrm{~d} \theta=1
$$

To calculate the reconstruction kernel for the Gaussian mollifier

$$
e_{\gamma}(\theta)=\frac{1}{c(\gamma)} \exp \left\{-\frac{\sin ^{2} \theta}{\gamma^{2}}\right\}, \quad \theta \in[0, \pi / 2],
$$

we need the following lemma, which is proved by a straightforward calculation.

Lemma 9. For integer $k$, nonnegative integer $n$, and $t>0$, it holds that

$$
\left(\frac{1}{t} \frac{\mathrm{d}}{\mathrm{d} t}\right)^{n} t^{k}=c_{k, n} t^{k-2 n}
$$

where $c_{k, n}=\prod_{j=0}^{n-1}(k-2 j)$.

Theorem 9. For the Gaussian mollifier in dimensions $d \geq 3$, the reconstruction kernel for the spherical Radon transform is given by

$$
\psi_{\gamma}^{R}(\theta)=\frac{B((d-1) / 2,(d-2) / 2)}{2(d-3) ! c(\gamma)} \sum_{k=0}^{\infty}\left(\prod_{r=0}^{k-1} \frac{(d-1) / 2+r}{d-3 / 2+r}\right) \frac{(-1)^{k} c_{2 d+2 k-5, d-2}}{k ! \gamma^{2 k}}(\cos \theta)^{2 k},
$$

where the $c_{k, n}$ are as in Lemma 9 and $B(\cdot, \cdot)$ denotes the beta function. The normalizing constant $c(\gamma)$ can be calculated using (15).

Proof. According to (14) we have

$$
\psi_{\gamma}^{R}(\arccos t)=\frac{1}{(d-3) ! c(\gamma)} t\left(\frac{1}{t} \frac{\mathrm{d}}{\mathrm{d} t}\right)^{d-2} \int_{0}^{t} \mathrm{e}^{-x^{2} / \gamma^{2}} x^{d-2}\left(t^{2}-x^{2}\right)^{(d-4) / 2} \mathrm{~d} x
$$

We begin with

$$
\begin{aligned}
\int_{0}^{t} \mathrm{e}^{-x^{2} / \gamma^{2}} x^{d-2}\left(t^{2}-x^{2}\right)^{(d-4) / 2} \mathrm{~d} x & \stackrel{x=s t}{=} t \int_{0}^{1} \mathrm{e}^{-(s t)^{2} / \gamma^{2}}(s t)^{d-2}\left(t^{2}-(s t)^{2}\right)^{(d-4) / 2} \mathrm{~d} s \\
& \stackrel{u=s^{2}}{=} \frac{1}{2} t^{2 d-5} \int_{0}^{1} \mathrm{e}^{-u t^{2} / \gamma^{2}} u^{(d-3) / 2}(1-u)^{(d-4) / 2} \mathrm{~d} u,
\end{aligned}
$$

where the integral is $B((d-1) / 2,(d-2) / 2)$ times the moment generating function of a $\operatorname{Beta}((d-1) / 2,(d-2) / 2)$-distributed random variable evaluated at $-t^{2} / 2 \gamma^{2}$. Thus, we obtain (see [6, p. 218]), for $X \sim \operatorname{Beta}((d-1) / 2,(d-2) / 2)$,

$$
\begin{array}{rl}
\frac{1}{2} t^{2 d-5} & B\left(\frac{d-1}{2}, \frac{d-2}{2}\right) m_{X}\left(\frac{-t^{2}}{\gamma^{2}}\right) \\
= & \frac{1}{2} B\left(\frac{d-1}{2}, \frac{d-2}{2}\right) \sum_{k=0}^{\infty}\left(\prod_{r=0}^{k-1} \frac{(d-1) / 2+r}{d-3 / 2+r}\right) \frac{(-1)^{k} t^{2 d+2 k-5}}{k ! \gamma^{2 k}} .
\end{array}
$$


With Lemma 9, this leads to

$$
\begin{aligned}
\psi_{\gamma}^{R}(\arccos t)=\frac{B((d-1) / 2,(d-2) / 2)}{2(d-3) ! c(\gamma)} t \sum_{k=0}^{\infty}\left(\prod_{r=0}^{k-1} \frac{(d-1) / 2+r}{d-3 / 2+r}\right) \\
\times \frac{(-1)^{k}((1 / t) \mathrm{d} / \mathrm{d} t)^{d-2} t^{2 d+2 k-5}}{k ! \gamma^{2 k}} \\
=\frac{B((d-1) / 2,(d-2) / 2)}{2(d-3) ! c(\gamma)} \sum_{k=0}^{\infty}\left(\prod_{r=0}^{k-1} \frac{(d-1) / 2+r}{d-3 / 2+r}\right) \frac{(-1)^{k} c_{2 d+2 k-5, d-2} t^{2 k}}{k ! \gamma^{2 k}} .
\end{aligned}
$$

Corollary 3. For the Gaussian mollifier, the reconstruction kernel for the cosine transform is given by

$$
\psi_{\gamma}(\theta)=\frac{1}{2 \omega_{d-1}}\left(\frac{1}{\sin ^{d-2} \theta} \frac{\partial}{\partial \theta}\left(\sin ^{d-2} \theta \frac{\partial \psi_{\gamma}^{R}(\theta)}{\partial \theta}\right)+(d-1) \psi_{\gamma}^{R}(\theta)\right),
$$

where $\psi_{\gamma}^{R}(\theta)$ is the reconstruction kernel for the Radon transform from Theorem 9.

Proof. For the block operator, defined by

$$
\square=\frac{\Delta_{d-1}+d-1}{2 \omega_{d-1}},
$$

where $\Delta_{d-1}$ is the Beltrami-Laplace operator on $S^{d-1}$, it holds that $C^{-1}=\square R^{-1}$ (see [18]). Since the Beltrami-Laplace operator on the sphere can be written using spherical coordinates, i.e.

$$
\Delta_{d-1}=\frac{1}{\sin ^{d-2} \theta} \frac{\partial}{\partial \theta}\left(\sin ^{d-2} \theta \frac{\partial}{\partial \theta}\right)
$$

for rotationally symmetric functions, this yields the result.

\section{Acknowledgements}

We would like to thank Evgeny Spodarev, Alfred K. Louis, and Zakhar Kabluchko for fruitful discussions and the anonymous referees for their useful comments. Our research has been supported by a grant from the German Research Foundation (DFG).

\section{References}

[1] Baddeley, A. And Vedel Jensen, E. B. (2005). Stereology for Statisticians (Monogr. Statist. Appl. Prob. 103). Chapman \& Hall/CRC, Boca Raton, FL.

[2] Den Hollander, F. (2000). Large Deviations (Fields Inst. Monogr. 14). American Mathematical Society, Providence, RI.

[3] Gardner, R. J. (2006). Geometric Tomography (Encyclopedia Math. Appl. 58), 2nd edn. Cambridge University Press.

[4] Gardner, R. J., Kiderlen, M. and Milanfar, P. (2006). Convergence of algorithms for reconstructing convex bodies and directional measures. Ann. Statist. 34, 1331-1374.

[5] Hardin, R. H. and Sloane, N. J. A. (1996). McLaren's improved snub cube and other new spherical designs in three dimensions. Discrete Comput. Geom. 15, 429-441.

[6] Johnson, N. L., Kotz, S. and Balakrishnan, N. (1995). Continuous Univariate Distributions, Vol. 2, 2nd edn. John Wiley, New York.

[7] Kiderlen, M. and Pfrang, A. (2005). Algorithms to estimate the rose of directions of a spatial fiber system. J. Microsc. 219, 50-60. 
[8] Korolev, V. and Shevtsova, I. (2012). An improvement of the Berry-Esseen inequality with applications to Poisson and mixed Poisson random sums. Scand. Actuarial J. 2012, 81-105.

[9] LouIs, A. K. AND MAAß, P. (1990). A mollifier method for linear operator equations of the first kind. Inverse Problems 6, 427-440.

[10] Louis, A. K., Riplinger, M., Spiess, M. And SPodarev, E. (2011). Inversion algorithms for the spherical Radon and cosine transform. Inverse Problems 27, 035015, 25pp.

[11] Mita, H. (1997). Probabilities of large deviations for sums of random number of i.i.d. random variables and its application to a compound Poisson process. Tokyo J. Math. 20, 353-364.

[12] Rubin, B. (2002). Inversion formulas for the spherical Radon transform and the generalized cosine transform. Adv. Appl. Math. 29, 471-497.

[13] Schladitz, K. (2000). Estimation of the intensity of stationary flat processes. Adv. Appl. Prob. 32, 114-139.

[14] SchneIder, R. AND WeIL, W. (2008). Stochastic and Integral Geometry. Springer, Berlin.

[15] Schuster, T. (2007). The Method of Approximate Inverse: Theory and Applications (Lecture Notes Math. 1906). Springer, Berlin.

[16] Sloan, I. H. AND Womersley, R. S. (2004). Extremal systems of points and numerical integration on the sphere. Adv. Comput. Math. 21, 107-125.

[17] Spiess, M. and Spodarev, E. (2011). Anisotropic Poisson processes of cylinders. Methodology Comput. Appl. Prob. 13, 801-819.

[18] Spodarev, E. (2001). On the rose of intersections of stationary flat processes. Adv. Appl. Prob. 33, 584-599.

[19] Stoyan, D., Kendall, W. S. And Mecke, J. (1995). Stochastic Geometry and Its Applications, 2nd edn. John Wiley, Chichester.

[20] WeIL, W. (1987). Point processes of cylinders, particles and flats. Acta Appl. Math. 9, 103-136. 